\title{
Lipid Interactions Between Flaviviruses and Mosquito Vectors
}

\section{OPEN ACCESS}

Edited by:

Hester Gertruida O'Neill, University of the Free State,

South Africa

Reviewed by:

Rushika Perera,

Colorado State University,

United States

Katia C. Gondim,

Federal University of Rio de Janeiro,

Brazi

*Correspondence:

Thomas Vial

Othomas.via@gmail.com

Julien Pompon

julien.pompon@ird.fr

${ }^{\dagger}$ Present address:

Thomas Vial,

Division of Virology, Department of Pathology, University of Cambridge,

Cambridge, United Kingdom: Department of Medical Microbiology,

Amsterdam UMC, University of Amsterdam, Amsterdam Netherlands

Specialty section: This article was submitted to Lipid and Fatty Acid Research, a section of the journal

Frontiers in Physiology

Received: 23 August 2021 Accepted: 20 September 2021 Published: 05 November 2021

Citation:

Vial T, Marti G, Missé $D$ and Pompon J (2021) Lipid Interactions Between Flaviviruses and Mosquito Vectors. Front. Physiol. 12:763195. doi: 10.3389/fphys.2021.763195

\author{
Thomas Vial ${ }^{1,2 *+}$, Guillaume Marti3,4, Dorothée Missé ${ }^{5}$ and Julien Pompon ${ }^{5 *}$ \\ 'Programme in Emerging Infectious Diseases, Duke-NUS Medical School, Singapore, Singapore, ${ }^{2}$ UMR 152 \\ PHARMADEV-IRD, Université Paul Sabatier, Toulouse, France, ${ }^{3}$ LRSV (UMR 5546), CNRS, Université de Toulouse, \\ Toulouse, France, ${ }^{4}$ MetaboHUB, National Infrastructure of Metabolomics and Fluxomics, Toulouse, France, ${ }^{5}$ MIVEGEC, \\ Université Montpellier, IRD, CNRS, Montpellier, France
}

Mosquito-borne flaviviruses, such as dengue (DENV), Zika (ZIKV), yellow fever (YFV), West Nile (WNV), and Japanese encephalitis (JEV) viruses, threaten a large part of the human populations. In absence of therapeutics and effective vaccines against each flaviviruses, targeting viral metabolic requirements in mosquitoes may hold the key to new intervention strategies. Development of metabolomics in the last decade opened a new field of research: mosquito metabolomics. It is now clear that flaviviruses rely on mosquito lipids, especially phospholipids, for their cellular cycle and propagation. Here, we review the biosyntheses of, biochemical properties of and flaviviral interactions with mosquito phospholipids. Phospholipids are structural lipids with a polar headgroup and apolar acyl chains, enabling the formation of lipid bilayer that form plasmaand endomembranes. Phospholipids are mostly synthesized through the de novo pathway and remodeling cycle. Variations in headgroup and acyl chains influence phospholipid physicochemical properties and consequently the membrane behavior. Flaviviruses interact with cellular membranes at every step of their cellular cycle. Recent evidence demonstrates that flaviviruses reconfigure the phospholipidome in mosquitoes by regulating phospholipid syntheses to increase virus multiplication. Identifying the phospholipids involved and understanding how flaviviruses regulate these in mosquitoes is required to design new interventions.

Keywords: mosquito, flavivirus, phospholipids, transmission, metabolomics

\section{MOSQUITO-TRANSMITTED FLAVIVIRUSES}

\section{Global Pathogens}

Flaviviruses like dengue (DENV), Zika (ZIKV), yellow fever (YFV), West Nile (WNV), and Japanese encephalitis (JEV) viruses threaten almost the whole human population (Pierson and Diamond, 2020). They cause half a billion infections per year that result in about 250,000 deaths and an economic loss of $\$ 8.9$ billion. All these viruses are transmitted through the bite of mosquito vectors, which geographic distributions are steadily increasing because of global changes. As a result, these diseases that were once restricted to the tropics have now encroached on temperate regions, encompassing new immunologically naïve populations. More alarmingly, the emergence of ZIKV and WNV in the last decades suggests that one should expect other flaviviruses to emerge as epidemic in the near future (Pierson and Diamond, 2020). 
There is no effective means to control all flaviviruses (Barrows et al., 2018). Rare examples of sustained vector control showed its ineffectiveness to prevent epidemics, even in small isolated urbanized areas (Ooi et al., 2006). The design of safe vaccines is challenging. Indeed, some antibodies generated during a primary flavivirus infection cross-react with a secondary heterologous flavivirus infection but fail to neutralize the second infection. These cross-reacting non-neutralizing antibodies then facilitate attachment and cell entry of the second virus, increasing infection and aggravating symptoms (Halstead, 2007) (a phenomenon called Antibody-Dependent Enhancement, ADE). The only licensed vaccine against DENV (i.e., DENGVAXIA) suffers from this limitation as it can increase dengue symptom severity in dengue-naïve patients (Halstead, 2017; Shukla et al., 2020; Huang et al., 2021). Moreover, there is no therapeutics and treatments are limited to supportive care.

Recent technical developments enabled the study of metabolic interactions in flavivirus infection [methodology of metabolomics was extensively reviewed in Nunes and Canuto (2020)]. Multiple evidence demonstrates the roles of lipids in infections in both vertebrate hosts and vectors (Leier et al., 2018; Byers et al., 2019; O'Neal et al., 2020), although the molecular mechanisms that orchestrate the lipidome modulations remain mostly elusive. Characterized lipid factors are potential targets for new mosquito-targeted interventions (Lu and Chen, 2017; Manchester and Anand, 2017). In this review, we focus on the biochemistry and functions of lipid metabolism in flavivirus infection in mosquito vectors.

\section{Flaviviruses}

Mosquito-transmitted flaviviruses such as the four DENV serotypes (DENV 1-4), ZIKV, YFV, JEV, and WNV are genetically related and primarily transmitted by either Aedes or Culex mosquitoes (Alkan et al., 2015; Lazear and Diamond, 2016; Figure 1). Flaviviruses are enveloped viruses with a spherical shape of about $50 \mathrm{~nm}$ diameter. The envelope is composed of two proteins, the Envelope and Membrane, which are anchored in a lipid bilayer. Within the envelope structure, a single-stranded positive-sense RNA genome of about $11 \mathrm{~kb}$ is packaged in association with the capsid protein. The genome codes for three structural proteins - Envelope, pr-Membrane, and Capsid - and seven non-structural proteins - NS1, NS2A, NS2B, NS3, NS4A, NS4B, and NS5. In the virion, the lipid bilayer is the only component that is not encoded in the viral genome and derives from the vector.

\section{Mosquito Transmission}

Viruses collected during a blood meal on an infected host first infect the mosquito midgut epithelium (Salazar et al., 2007). While blood meal is digested in $48 \mathrm{~h}$, viral replication in the midgut continues and reaches a peak at 7 days after blood ingestion. From the midgut, viruses disseminate in the whole mosquito body, including salivary glands which are fully infected at 10-14 days post infectious blood meal. Mosquitoes can then transmit the viruses via expectorated saliva during subsequent bites.
Throughout infection in mosquito, flaviviruses are confronted to different barriers (Franz et al., 2015). After ingestion with blood, flaviviruses need to overpass an extracellular matrix to infect the midgut and then escape from the midgut to infect secondary tissues, such as hemocytes, fat body, and nerve tissues (Parikh et al., 2009). Viruses eventually infect lateral and median lobes of salivary glands (Salazar et al., 2007), which produce viral particles released in the excretory canal during biting. The ability of a mosquito to acquire and propagate viruses through all these steps is defined as vector competence. Vector competence is related to physical tissue barriers and genetic factors which, among others, determine immune response (Hardy et al., 1983). Because of the virus requirements for lipids, cellular lipid composition is another important factor that determines vector competence.

\section{PHOSPHOLIPID: A STRUCTURAL LIPID ON THE FRONTLINE}

\section{Phospholipid Definition}

Phospholipids (PL) are composed of one hydrophilic head group, a glycerol backbone and two hydrophobic fatty acyl chains (Figure 2A). Depending on their head group, they are categorized as phosphatidic acid (PA), phosphatidylcholine (PC), phosphatidylethanolamine (PE), phosphatidylserine (PS), phosphatidylglycerol (PG), cardiolipin (CL), or phosphatidylinositol (PI; van Meer et al., 2008; Figure 2B). The amphiphilic (i.e., having both hydrophilic and hydrophobic ends) nature of PL enables the formation of bilayers with the hydrophobic acyl chain turned inward. PLs are the major structural lipids and generate physical barriers circumventing cellular contents and segregating cytosolic compartments, enabling intracellular organelle formation and compartmentalization of different cellular activities (Vance, 2015).

Phospholipid biosynthesis is intimately connected with the tricarboxylic (TCA) cycle and glycolysis (Figure 2C). Carbohydrate catabolism during glycolysis, fatty acids oxidation and amino acid recycling produce acetyl-CoA. Acetyl-CoA then serves as a precursor for biosynthesis of fatty acids via lipogenesis. Acetyl-CoA also feeds in the TCA cycle to produce precursors of amino acids and the reducing agent $\mathrm{NADH}$, which goes into the electron transport chain to produce chemical energy in the form of ATP. The pentose phosphate pathway derived from glycolysis generates nucleotides and nucleic acids. All the general metabolic pathways are interconnected.

\section{Fatty Acid Biosynthesis}

The fatty acyl chains of PL can have different carbon number and degree of saturation, both of which are generated during fatty acid biosynthesis. Fatty acid synthesis starts with the formation of malonyl-CoA from acetyl-CoA, via acetyl-CoA carboxylase (Sul and Smith, 2008). In the cytoplasm, fatty acid synthase then catalyzes repeated additions of acetyl-CoA to mainly produce palmitic acid (palmitate), a 16-carbon saturated fatty acid (C16:0), and in minor amount, a 18-carbon stearic acid 
FIGURE 1 | Phylogenetic distribution of mosquito-borne flaviviruses and their associated vectors. Dendrogram based on amino acid sequences.

(C18:0) (Figure 3A). Palmitic or stearic acids undergo elongation or unsaturation to generate other types of fatty acids, such as oleic acid, linoleic acid, or arachidonic acid (Figure 3A; Cook and McMaster, 2002). Chain elongation is happening mainly in the endoplasmic reticulum (ER) and produces acyl chains greater than 16 carbons, by successive 2-carbon condensation from malonyl-CoA. Mitochondrial elongation is less important but is required for mitochondrial membrane biogenesis. Fatty acids are used as components of membrane lipids or can be esterified in triacylglycerol for energy storage.

Mono-unsaturated fatty acids are produced by oxidative desaturation by desaturases to form a double bond. The first double bond introduced is generally in carbon at position 9 $(\Delta 9)$ (Figure 3B). In most mammals, poly-unsaturated fatty acids result from the insertion of other double bonds between the existing bond and the carboxyl end of the chain, but not on the methyl end (Figure 3B; Rivers et al., 1975). In humans, polyunsaturated fatty acids, such as arachidonic acid, are synthetized through modification of linoleic acid via a $\Delta 6 / \Delta 5$ desaturase, followed by elongation steps (Bond et al., 2016). Insects, however, can desaturate on either side of the existing bond in $\Delta 9$ thanks to specific desaturases (Cook and McMaster, 2002). As a result, insect poly-unsaturated fatty acids contain double-bonds in positions $\Delta 5$ to $\Delta 15$, whereas mammal fatty acids can only produce direct double-bonds in positions $\Delta 4$ to $\Delta 9$ (Figure $3 B$ ).

\section{De novo Phospholipid Biogenesis}

Phospholipid biogenesis is highly conserved throughout the animal kingdom. It involves multiple enzymes in different organelles and results in the production of hundreds of different PL species (Figure 4). PL de novo biogenesis is initiated by two types of acyl-transferases that sequentially add two acyls to one glycerol-3-phosphate (Fagone and Jackowski, 2009; Takeuchi and Reue, 2009; Vance, 2015). The first addition is realized in the ER or mitochondria-associated membranes by glycerol-3-phosphate acyltransferase (GPAT) to produce lysoPA. LysoPAs produced in mitochondria are then transferred to the ER prior to the second acylation. The second addition is catalyzed by 1-acyl-sn-glycerol-3-phosphate O-acyltransferases (AGPAT) that transform lysoPA in PA in the ER principally (Yamashita et al., 2014). The new PA is composed of fatty acids linked to the glycerol molecule at the first and second carbons, called sn 1 and sn2 positions, respectively. Fatty acids in sn 1 are generally saturated or monounsaturated, while fatty acids in sn2 are polyunsaturated with longer chains (Beermann et al., 2005). The most abundant fatty acids in sn 1 are palmitic acid (16:0), stearic acid (18:0), and oleic acid (18:1); and in sn2 are linoleic acid (18:2), arachidonic acid (20:4), eicosapentaenoic acid (20:5), or docosahexaenoic acid (22:6) (Figure 3A). The diversity of fatty acids results in a multiplicity of PA species, which are then used to produce all PLs. Due to their implication in generating PA, GPATs are rate-limiting enzymes in PL biogenesis (Yamashita et al., 2014).

De novo PL biogenesis then separates in two branches, producing either diacylglycerol (DAG) or cytidine diphosphate (CDP)-DAG (Figure 4). Synthesis of CDP-DAG involves condensation of cytidine triphosphate via CDP-DAG synthase. This reaction occurs in ER and mitochondria-associated membranes. CDP-DAG is used to produce PI and PG. PGs are synthesized in both ER and mitochondria by phosphatidylglycerol phosphate synthase and phosphatase. PG can produce CL by combination with CDP-DAG via cardiolipin synthase or bis(monoacylglycerol)phosphate via a complex biosynthetic pathway of acylations. PI is synthesized by condensation of CDP-DAG with inositol via PI synthase. Inositol originates from diet, recycling, or biosynthesis from glucose. PI is the precursor of several phosphorylated derivatives, also called phosphoinositides, which are involved in cell signaling.

Phosphatidic acid is also transformed in DAG by PA phosphatase (Figure 4). DAG produces triacylglycerol and 


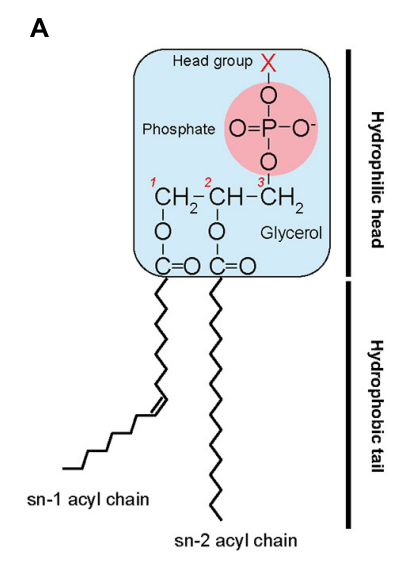

B
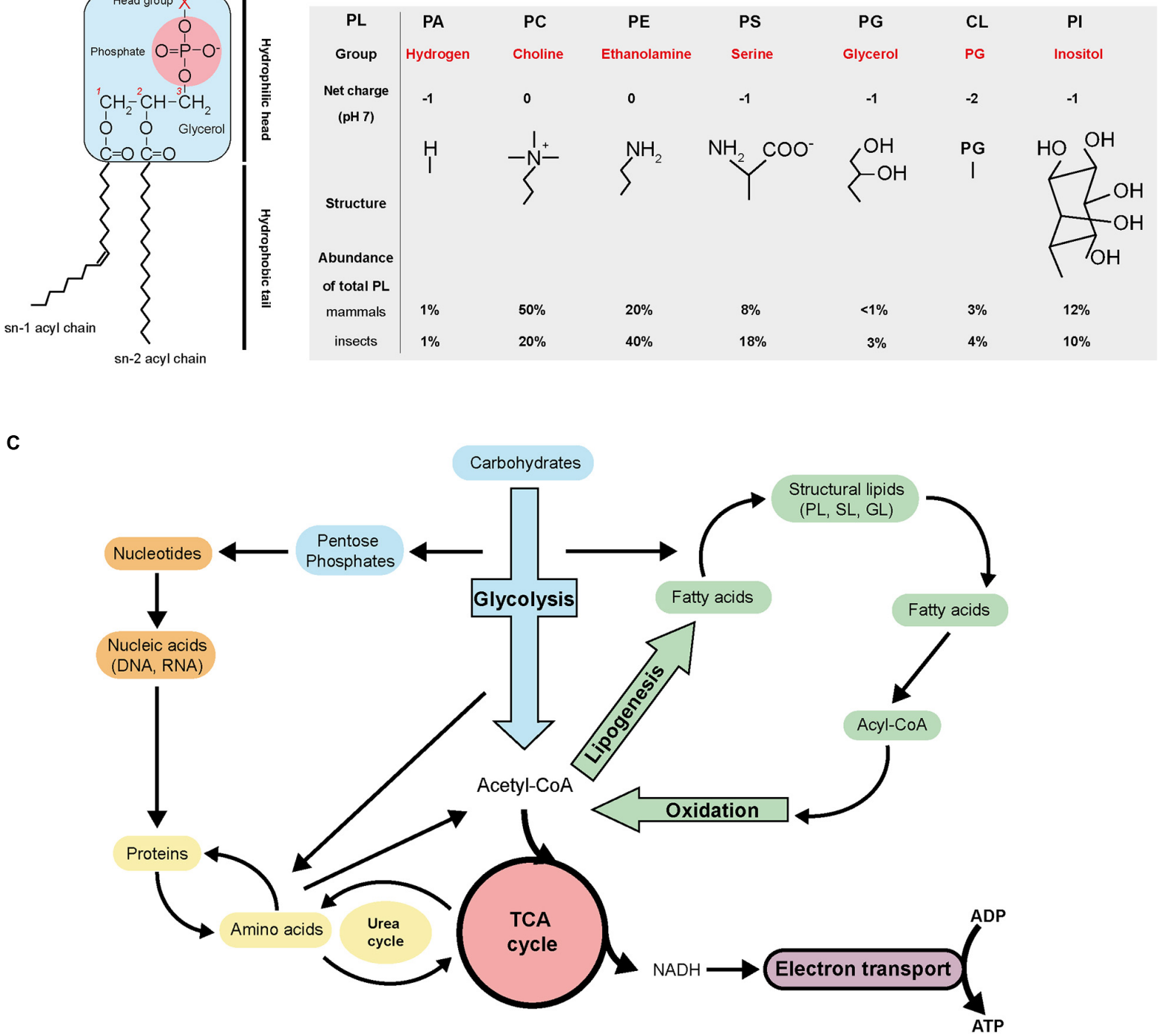

FIGURE 2 | Phospholipid structure and general metabolism. (A) Schematic of phospholipid structure. (B) Basic characteristics of the different phospholipid categories. (C) Overview of the general metabolism. PL, phospholipid; PA, phosphatidic acid; PC, phosphatidylcholine; PE, phosphatidylethanolamine; PS, phosphatidylserine; PG, phosphatidylglycerol; CL, cardiolipin; PI, phosphatidylinositol; TCA, tricarboxylic cycle; SL, sphingolipid; GL, glycerolipid; NADH, nicotinamide adenine dinucleotide; ADP, adenosine diphosphate; ATP, adenosine triphosphate.

feeds into the biosynthesis of aminophospholipids (aminoPL), namely PC, PE, and PS (Vance, 2015). De novo PC and PE synthesis is conducted through the Kennedy pathway (Gibellini and Smith, 2010) within the ER, using CDP-choline and CDP-ethanolamine intermediates. Choline and ethanolamine are first phosphorylated by choline/ethanolamine kinases (CK/EK). Phosphocholine and phosphoethanolamine then form CDP-choline and CDP-ethanolamine by combining with cytidine triphosphate through the rate-limiting enzyme cytidine triphosphate:phosphocholine/ethanolamine cytidylyltransferase (CT/ET). DAG incorporates the phosphocholine/phosphoethanolamine group from CDPcholine or CDP-ethanolamine with DAG:CDP-choline cholinephosphotransferase (CPT) or DAG:CDP-ethanolamine ethanolaminephosphotransferase (EPT) to produce PC and PE, respectively. Alternative pathways exist for $\mathrm{PC}$ and $\mathrm{PE}$ syntheses. PE biosynthesis through the PS decarboxylase (PSD) pathway in the mitochondria inner membrane involves PS decarboxylation into PE by a PS decarboxylase (Horvath et al., 2012; Tamura et al., 2012). PC can also be synthetized in a minor pathway via ethanolamine methylation of $\mathrm{PE}$ by $\mathrm{PE}$ methyltransferase, principally in hepatocytes. PC and PE are used to produce PS by head exchange reaction catalyzed by PS synthases (PSS1 or PSS2). Direct PS biosynthesis via CDP-DAG pathway was found in plants and yeast (Yamashita and Nikawa, 1997). PCs are also used in sphingomyelin synthesis by sphingomyelin synthase (SMS).

Given their roles in aminoPL biosynthesis, choline and ethanolamine are essential nutrients that need to be imported 
A

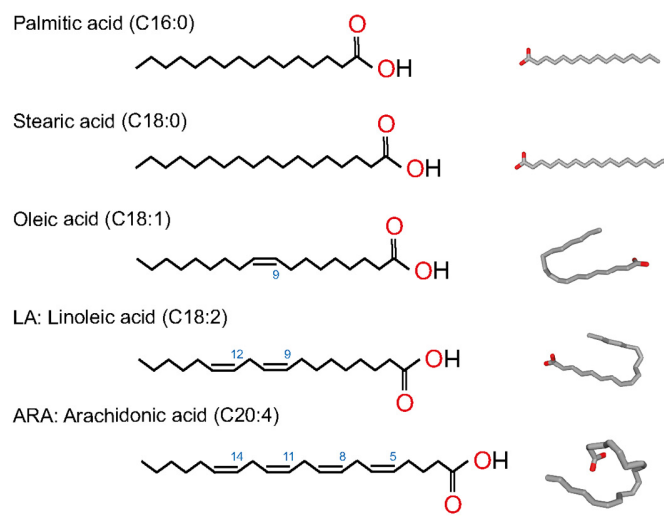

B

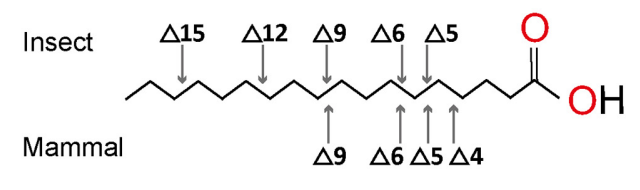

FIGURE 3 | Structures of most common fatty acids found in phospholipids. (A) Fatty acids saturated and unsaturated are represented in linear form and 2D conformation from https://pubchem.ncbi.nlm.nih.gov. (B) Desaturation positions in fatty acyls in insects and mammals.

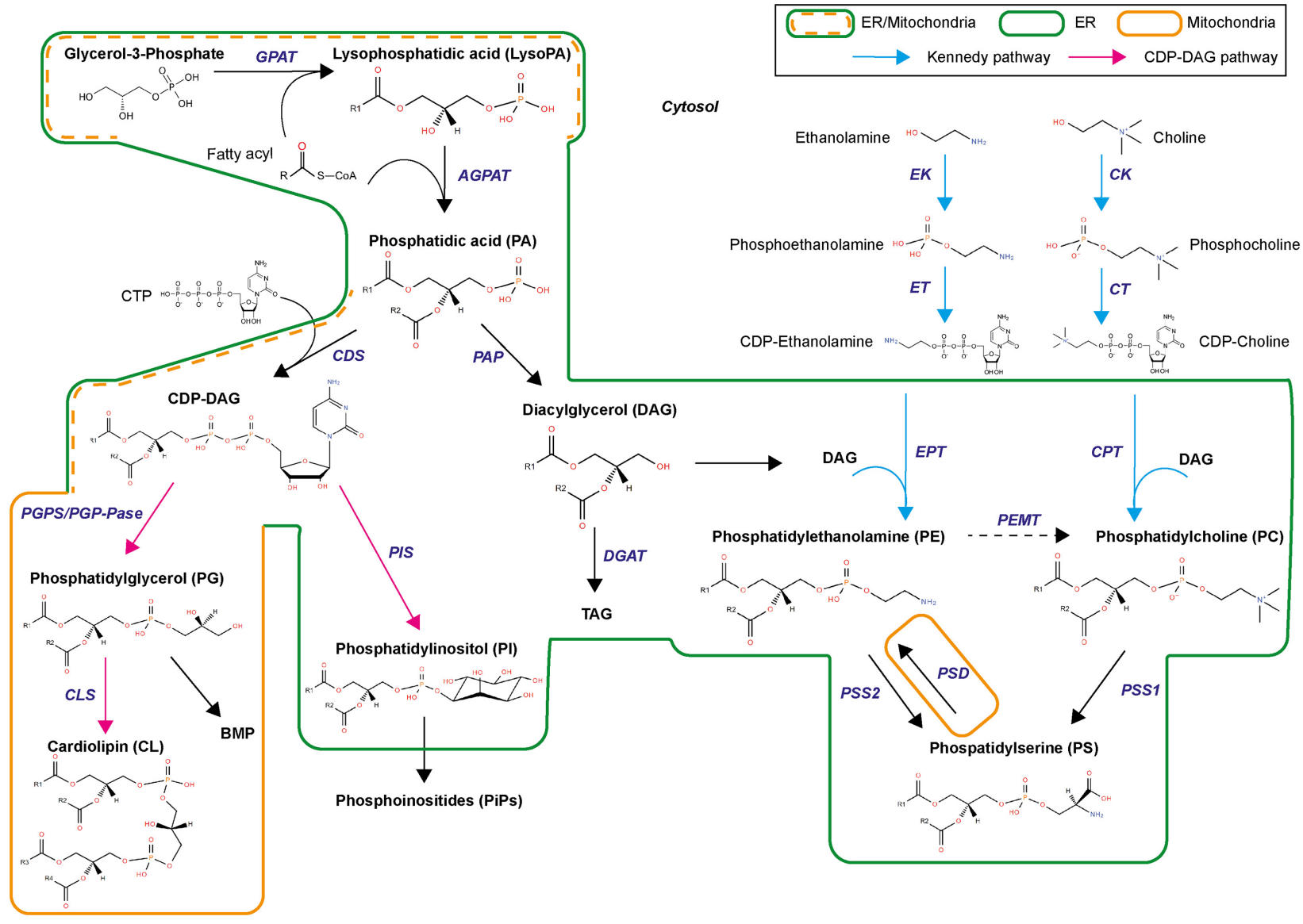

FIGURE 4 | Biosynthetic pathways for phospholipids. GPAT, glycerol-3-phosphate acyltransferase; AGPAT, 1-acyl-sn-glycerol-3-phosphate O-acyltransferases; CDS, CDP-diacylglycerol synthase; PGP, PG phosphate synthase, CLS, cardiolipin synthase, PIS, PI synthase; PAP, PA phosphatase; DGAT, Diglyceride acyltransferase; EK, ethanolamine kinase; CK, choline kinase; ET, triphosphate:phosphoethanolamine cytidylyltransferase; CT, triphosphate:phosphocholine cytidylyltransferase; EPT, DAG:CDP-ethanolamine ethanolaminephosphotransferase; CPT, DAG:CDP-choline cholinephosphotransferase; PEMT, PE methyltransferase; PSD, PS decarboxylase; PSS1, PS synthase 1; PSS2, PS synthase 2. 
from the diet (Gottlieb et al., 1999). Alternatively, minor mechanisms of recycling exist (Vance et al., 2007; Vance and Vance, 2008; Vance, 2013). Methylation of PE into PC followed by hydrolysis can recycle choline, while small amount of ethanolamine can be produced by PE degradation. Intracellularly, although choline and ethanolamine are rapidly phosphorylated, incorporation into CDP is a limiting reaction (Wortmann and Mayr, 2019). Consequently, phosphocholine and phosphoethanolamine are in higher quantities than the product from the following step, CDP-choline and CDP-ethanolamine. There exist feed-back and feed-forward mechanisms to adjust aminoPL production based on PL requirements (Patton-Vogt and de Kroon, 2020).

\section{Phospholipid Remodeling}

Phospholipid remodeling occurs through the Lands cycle and reconfigures fatty acyls in PLs, thereby increasing diversity of fatty acyls in de novo PLs (Wang and Tontonoz, 2019; Figure 5A). PLs are first hydrolyzed at the sn2 position by phospholipase A2 (PLA2) to produce 1-acyl lysoPL (Figure 5B). The lysoPL is then reacylated by lysophospholipid acyltransferase (LPLAT) via incorporation of another fatty acid in sn 2 position, thereby forming a new PL species (Wang and Tontonoz, 2019). Remodeling enables modulation of PL membrane composition and cell signaling. For the latter, lysoPL and fatty acids released by PLA2 activity can serve as intermediates for synthesis of signaling lipids (O’Donnell et al., 2018).

Phospholipase A2 enzymes have multiple isoforms with different mechanisms of action (Burke and Dennis, 2009). Four main categories of PLA2 exist (Nor Aliza and Stanley, 1998; Abdul Rahim et al., 2018): (i) secreted PLA2, which is most studied in bees and snake venoms and found in pancreatic juices from mammals; (ii) cytosolic PLA2, which is recruited to the membrane by $\mathrm{Ca}^{2+}$-dependent translocation; (iii) calciumindependent PLA2; and (iv) platelet activating factor lipoproteinassociated PLA2. There exist other types of phospholipases that can hydrolyze PL (Figure 5B; Aloulou et al., 2018). PLA1 hydrolyzes the sn1 position to produce a fatty acid and a lysoPL. PLA1 function is largely unknown but likely related to production of lysoPS, lysoPI, and lysoPA. Phospholipase B (PLB) hydrolyzes both the sn1 and sn2 fatty acids of PC, PE, and PI (Morgan et al., 2004). Phospholipase C (PLC) and phospholipase D (PLD) are phosphodiesterase. PLC cleaves the glycerophosphate bond of PC and PI, producing DAG and a phosphorylated headgroup. PLD removes the headgroup to generate PA, but can also catalyze exchanges of headgroups by transphosphatidylation to produce new PL types. In mammals, PLD is involved in PA remodeling and can release choline when PCs are the substrate (Onono and Morris, 2020).

Among LPLAT (Figure 5A), lysophosphatidylcholine acyltransferases (LPCAT) were first discovered for catalyzing reacylation of lysoPC (Wang and Tontonoz, 2019). LPCATs actually have acyltransferase activity for lysoPE, lysoPS, and lysoPG. Four LPCATs were identified (LPCAT1-4) and each has specific substrate preference, enzymatic activity and tissue localization in mammals. LPCAT1 and LPCAT2 are members of the AGPAT family and are found in the ER membrane and lipid droplets. PL remodeling by LPCAT1-2 regulates the size and surface organization of lipid droplets (Moessinger et al., 2014). LPCAT3 and LPCAT4 are part of the membrane-bound $\mathrm{O}$-acyltransferase family and are present in the ER membrane. LPCAT3 is the most expressed LPCAT in several cell types and is responsible for the bulk of lysoPC acyltransferase activity.

\section{Phospholipid Cellular Distribution}

Endoplasmic reticulum is where the bulk of structural lipids (i.e., PL, ceramides, and cholesterols) are produced (Bell et al., 1981). As the first production organelle, the ER contains all intermediates and end products of complex lipid pathways, except for sterol and sphingolipids which are rapidly transported into other membranes. Mitochondria is also a major site of lipid biosynthesis, especially for lysoPA, PA, and PG used for CL synthesis, a product that is unique to this organelle. Mitochondria can produce PE by PS decarboxylation. The inner
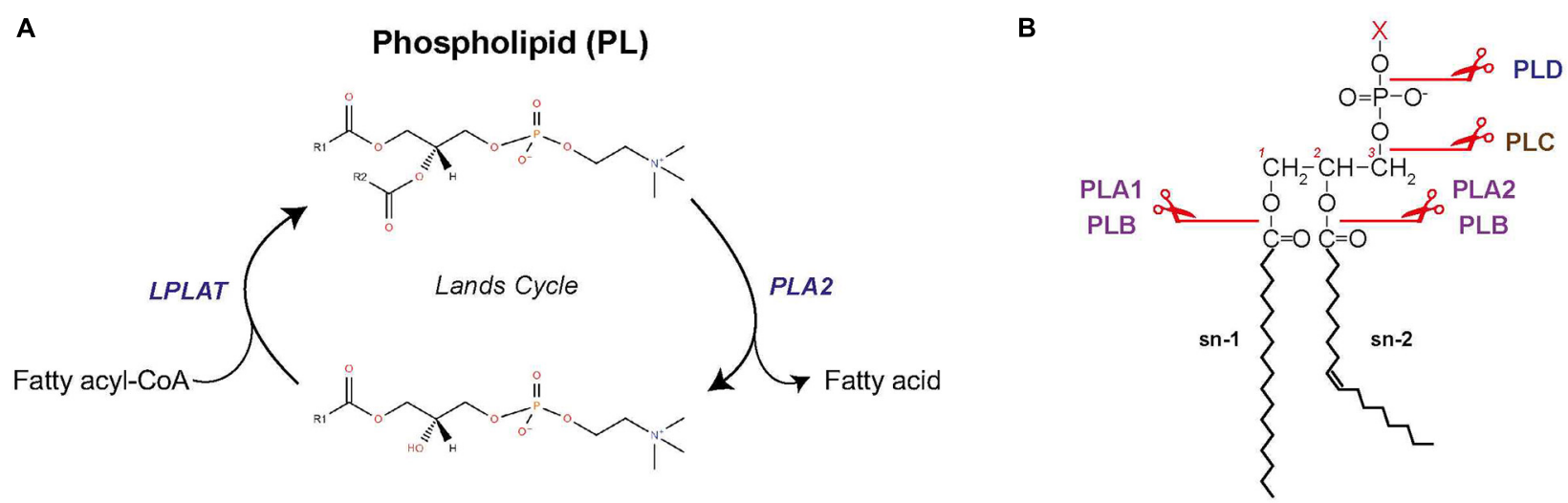

\section{Lysophospholipid (LysoPL)}

FIGURE 5 | Phospholipid remodeling. (A) Schematic of PL remodeling through the Lands cycle. (B) Sites of cleavage by the different phospholipases. LPLAT, Iysophospholipid acyltransferase; PLA1, phospholipase A1; PLA2, phospholipase A2; PLB, phospholipase B; PLC, phospholipase C; PLD, phospholipase D. 
membrane of the mitochondria is composed of a high PG and CL content and a high PE/PC ratio (Horvath and Daum, 2013). A sub-fraction of the ER attached to the mitochondria, the mitochondria-associated membranes, contains specific enzymes for lipid biosynthesis (Rusiñol et al., 1994; Raturi and Simmen, 2013; Mayr, 2015). The Golgi is more specialized in sphingolipid production and the final steps of PC synthesis (Henneberry et al., 2002). Plasma membranes and early endosome contain more sterols and sphingolipids than PLs, due to the required property of resistance to mechanical stress. Plasma membrane is not a major place for structural lipid synthesis, even if lipid regulation occurs by sphingolipid turnover and lipid degradation (Di Paolo and De Camilli, 2006). Late endosome contains low concentrations of PS and sterol but a high concentration of bis(monoacylglycerol)phosphate, a lipid associated with fusion and sphingolipid degradation (Kobayashi et al., 2002; Kolter and Sandhoff, 2005).

\section{Phospholipid Biochemical Properties Curvature of Membranes}

As steric hindrance of PL varies with the headgroup size and the acyl chains, PL composition influences membrane shape (Marsh, 2007; Marquardt et al., 2015; McMahon and Boucrot, 2015). PC and PS have a relatively large polar headgroup and parallel fatty acyl chains that confer a cylindrical geometry and enable linear bilayer formation (Figure 6A). Similarly, sphingolipids have a smaller headgroup and only one acyl chain, resulting in a cylindrical geometry. However, when inserted in bilayers, sphingolipids produce a tighter membrane because of their smaller steric hindrance. PE, PA, DAG, and CL have a small

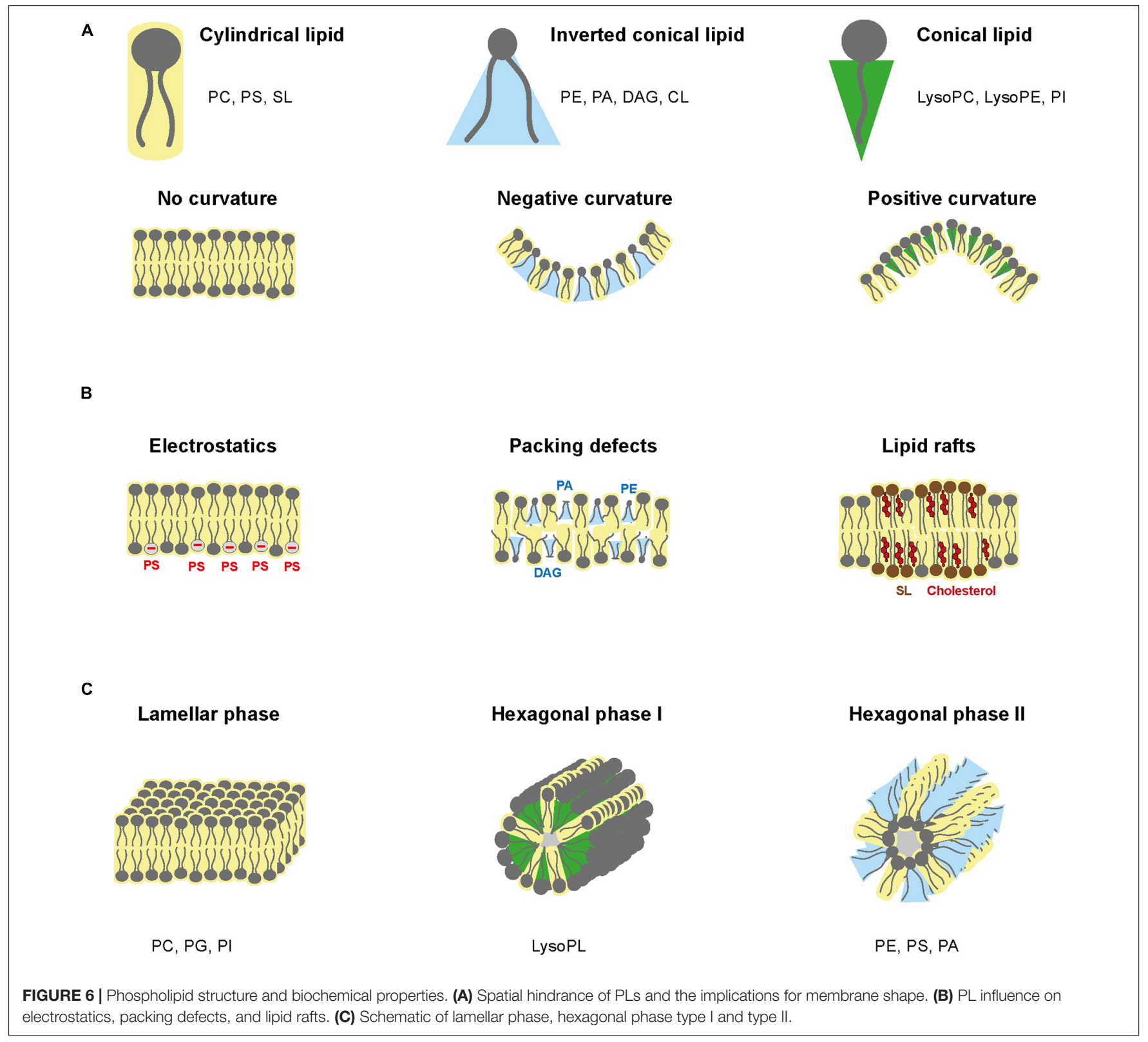


headgroup and two fatty acyls that form an inverted conical geometry (Figure 6A). When inserted in the inner layer of a lipid bilayer, these $\mathrm{PL}$ types impose a negative curvature (Marsh, 2007). Conversely, lysoPC, lysoPE, and PI have a thinner acyl chain hindrance, which confers positive curvature in lipid bilayer (Figure 6A).

\section{Membrane Asymmetry}

Membrane asymmetry refers to an asymmetric distribution of lipid species in lipid bilayers. Asymmetry induces biophysical properties that promote certain cellular functions (Fadeel and Xue, 2009; Marquardt et al., 2015). A well-known example is the organization of negatively charged PS in plasma membrane. In normal cells, PS are found on the cytoplasmic side where they associate with numerous enzymes such as kinases. During specific events, PS move to the outer leaflet, exposing their negative polar head to the extracellular side and inducing phagocytosis. In plasma membrane, $\mathrm{PC}$ and sphingolipid are generally found in the outer leaflet, while PE, PS, and PI are found in the inner leaflet (van Meer et al., 2008). Lipids in the ER are mostly symmetrically distributed between the two leaflets, while Golgi and endosome membranes have asymmetric distributions.

Phospholipid asymmetry is maintained by lipid transporters (Leventis and Grinstein, 2010; Kobayashi and Menon, 2018). Flippases are ATP-dependent aminoPL translocases that transport lipids inward. Floppases are ATP-binding cassette transporters that transport lipids outward. PL scramblases induce lipid asymmetry by exchanging lipids between the two membrane layers. For instance, PL scramblases induce PS externalization in apoptotic cells (Fadeel and Xue, 2009; Williamson, 2016).

\section{Electrostatics}

Electrostatic charges of membranes depend on negatively charged lipids, i.e., PS and phosphoinositides (PIPs) (Figure 6B). PS and PIPs are highly present in plasma membrane, especially on the cytosolic side and are in low abundance in ER membrane (Holthuis and Levine, 2005; Leventis and Grinstein, 2010). Furthermore, the charge of other PLs is pH-dependent. PC and $\mathrm{PE}$ are zwitterionic, whereas PS, PA, PG, CL, and PI are anionic at $\mathrm{pH} 7$ (Li et al., 2015). Consequently, $\mathrm{pH}$ gradient modifies electric charges (Hope and Cullis, 1987). Electric charges are critical to orientate transmembrane proteins. Positively charged peptides will interact with negatively charged lipids in the inner leaflet to integrate into the lipid bilayer, while the protein position within the membrane is determined by charges from both lipid layers (Marquardt et al., 2015). Eventually, distribution of the membrane electric charges is influenced by lipid asymmetry.

\section{Packing Defects}

Lipid packing defects refer to heterogeneous lipid arrangements, which loosen lipid bilayer and increase fluidity, facilitating protein insertion (Janmey and Kinnunen, 2006). Ratio between small and large headgroups and ratio between saturated and unsaturated acyl chains influence lipid packing. Low packing in the ER is induced by high concentration of unsaturated PL and lack of cholesterol (Radhakrishnan et al., 2008), while plasma membrane has high packing due to saturated structural lipids and sterols (Figure 6B).

\section{Lipid Phases}

Lipid phase relates to the fluidity of lipid membranes and how lipids can move within each layer. The liquid phase is characterized by lateral mobility of lipids within their layer, whereas in the solid phase, also called gel phase, lipids lose lateral mobility. Lipid phase depends on the lipid composition and extrinsic factors such as temperature, pressure, and composition of the aqueous phase (van Meer et al., 2008). Most biomembranes are organized in lamellar (i.e., bilayer structure) with lipids in liquid phase and contain floating "rafts" of gel-phase lipids (Figure 6B). Lamellar structures are formed by non-curvature lipids such as PC, PG, and PI, while long and saturated chains, high amount of cholesterol and low temperature promote gel phase formation. The gel-phase rafts are enriched in saturated lipids but also sphingolipids and cholesterol, while the liquidphase contains unsaturated PLs, which form kinks that loosen interactions with other lipids.

Membrane lipids can also form non-lamellar transitory structures, known as hexagonal phases (Jouhet, 2013; Figure 6C). Hexagonal phase can be of two types. Type I hexagonal phase has the polar head outside of the micelles, whereas type II consists of inverted micelles with fatty acyl chains directed outward. Combination of type II and lamellar phases can establish aqueous channels within the lipid bilayer. Non-lamellar phases exist temporarily during fusion, fission and pore formation and influences specific biochemical reactions (Jouhet, 2013). Negative curvature lipids, such as PE, PS, and PA, form hexagonal type II micelle phase, while positive curvature lipids such as lysoPL form hexagonal type I phase.

\section{Protein Insertion}

Proteins inserted in membranes drastically modify the behavior of lipid membranes. Insertion of proteins is influenced by the physicochemical parameters of the membrane, such as curvature, electrostatics, and lipid packing (Bigay and Antonny, 2012). Once inserted, the proteins perturb the hydrophobicity by provoking a mismatch between protein and lipid that affects the thickness and membrane organization (Janmey and Kinnunen, 2006). Inversely, protein function is also tightly regulated by lipid interactions, which can influence its interaction with other molecules (Lundbæk, 2006; Martens et al., 2018).

\section{Lipid Droplets: A Lipid Storage in Interaction With Phospholipids}

Lipid droplets (LD) are essential for cell storage of carbons in the form of neutral lipids, triacylglycerol and sterol esters (Jackson, 2019). These storage bodies are constrained by a monolayer of PL (mainly PC and PE) and proteins (Bartz et al., 2007). LD are generated by neutral lipid accumulation (TAG and sterol esters) between the two ER phospholipid bilayers. Growing of this lipid lens produces a vesicle surrounded by a monolayer originating from the ER that is eventually released into the cytosol (Thiam and Forêt, 2016). LD biogenesis is tightly regulated by PL metabolism through GPAT, DGAT, and CPT enzymes, 
respectively, involved in LysoPA, TAG, and PC biosynthesis (Krahmer et al., 2011; Henne and Goodman, 2019). Other proteins not directly associated with lipid syntheses are involved in LD production and include seipin, perilipins and fat storageinducing transmembrane (FIT) proteins. Nonetheless, seipin can regulate PL and neutral lipid synthesis, as well as PL transfer between the ER and LD (Wang et al., 2016; Yan et al., 2018).

Lipids in LD are mobilized by lipases to provide substrates for PL synthesis, fatty acid pathway, and signaling lipid production (Ducharme and Bickel, 2008). LD also contain enzymes of the PC biosynthesis pathway, allowing the production of CDP-choline (Penno et al., 2013). However, LD-produced CDP-choline is then transferred to the ER to complete PC production. Presence of PLA2 and LPCAT in LD can also influence PL remodeling and the PL monolayer surrounding LD. While this review focuses on the role of LD in PL metabolism, it should be noted that LD have other functions in vitamin storage, vitamin signaling, regulation of cellular stress, protein metabolism and interact with several organelles (Welte and Gould, 2017).

\section{FLAVIVIRUS CELLULAR CYCLE IS INTRICATELY LINKED TO MEMBRANE LIPIDS}

\section{Lipid Composition of Virions}

Flavivirus virions are composed of three structural proteins, all of which interact with lipids. The envelope and membrane proteins form the outer layer of the virion and are anchored in a lipid bilayer membrane. The asymmetric charge distribution of the capsid enables interaction with lipid membranes ( $\mathrm{Ma}$ et al., 2004) and LDs, the latter being required for virus particle formation (Samsa et al., 2009). Lipid composition of flavivirus virions was only characterized for WNV (Martín-Acebes et al., 2014) and contained a majority of sphingolipids and PLs. Among PLs, PC was the most abundant type, followed by PS and plasmalogen-PC. PE, plasmalogen-PE, lysoPE, and lysoPC were also present but in lower quantities. While there is an abundance of cylindrical lipids such as sphingolipids and PC, integration of the transmembrane envelope and membrane proteins is likely responsible for the curvature that results in a spherical virus particle. Computational models of DENV lipid envelope indicate an important biophysical robustness, characterized by a slow lipid diffusion (Reddy and Sansom, 2016).

\section{Structural Lipids in Virus Attachment and Entry}

Virus attachment occurs through interactions with cell surface factors, including, but not restricted to, lipids (Figure 7). There exists a variety of receptors in mammalian and mosquito cells, consistent with the ability of flaviviruses to infect a diversity of cells from two different hosts (Hidari and Suzuki, 2011; Cruz-Oliveira et al., 2015). The T-cell immunoglobulin mucin protein domain 1 (TIM) and the tyrosine protein kinase receptor 3-AXL-MER (TAM) families of proteins act as ligands to PS and PE from the viral envelope and promote cell entry
(Meertens et al., 2012; Richard et al., 2015). Similarly, human CD300a is a PL receptor and binds directly to PE and PS from DENV particles (Carnec et al., 2016).

After virus adsorption to cell surface, entry occurs mainly by clathrin-dependent endocytosis (Figure 7). As compared to mammals, only clathrin-mediated endocytosis was observed for the four DENV serotypes in mosquito cells (Mosso et al., 2008; Acosta et al., 2011). An invagination in the plasma membrane encloses the virus in a clathrin-coated vesicle, which is transported inside the cell by a mechanism involving actin filaments (Acosta et al., 2008). During internalization, the vesicle acidification changes the envelope protein conformation and enables fusion of viral and endosomal membranes. The fusion process is PL-dependent, especially for PS and bis(monoacylglycero)phosphate (Zaitseva et al., 2010). The viral genome is eventually released as a ribonucleoprotein into the cytoplasm.

Cholesterol is a crucial lipid during flavivirus entry, playing a role both in the target cell and in the viral particle. Depletion of cellular cholesterol inhibits DENV and JEV entry (Lee et al., 2008), while supplementation of viral particles with cholesterol similarly blocks DENV entry (Carro and Damonte, 2013). Cholesterol is enriched in plasma membrane where it associates with PL and sphingolipids to form gel-phase rafts. Alteration of these rafts may influence presentation of attachment receptors or endocytosis.

\section{Endoplasmic Reticulum Membrane as a Platform for Translation}

Once released, the encapsidated genome is uncoated and recruited to ER-bound ribosomes for translation. The single open reading frame of the genome is translated into a multipass transmembrane polyprotein, which is processed by viral and host proteases into the three structural proteins (capsid, pr-Membrane, and Envelope) and seven non-structural proteins (NS1, NS2A, NS2B, NS3, NS4A, NS4B, and NS5) (Barrows et al., 2018; Figure 7). Successful folding and post-translational stability require the ER membrane protein complex (Lin et al., 2019; Ngo et al., 2019), sphingolipid and cholesterol-rich lipid rafts (García Cordero et al., 2014). The translation is intertwined with the ER membrane and several viral proteins remain anchored in the ER after translation. While translation and the transmembrane proteins should affect membrane topology and lipid composition, little is known about their impacts on PL reconfiguration.

\section{Endomembranes at the Heart of Genome Replication}

To initiate genome replication, a negative single-strand RNA antigenome is produced from the entering positive-strand genome and then used as a template to duplicate the positivestrand genome (Figure 7). Replication complexes formed by ER invaginations enable genome replication and isolation from host defenses. Structures of DENV replication complex have been characterized in human and mosquito cells (Welsch et al., 2009; Junjhon et al., 2014). In mammalian cells, membrane alterations form several structures: convoluted membranes, 


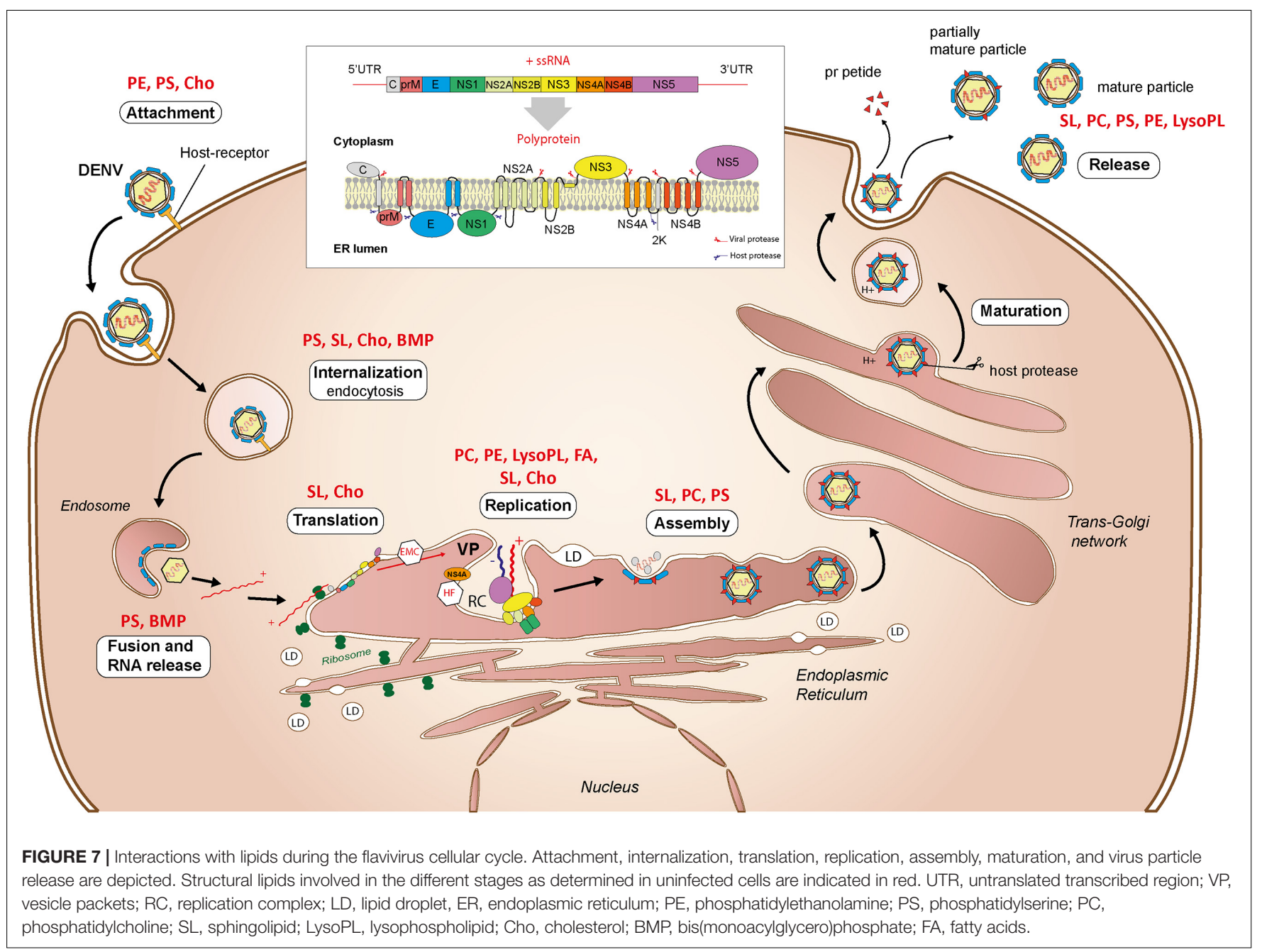

double-membrane vesicles, tubular structures, and vesicle packets. Except for convoluted membranes, all the distinct structures were observed in mosquito cells. These different structures are connected by pores, probably to enable transport of building blocks for RNA synthesis and/or release of newly synthetized RNA. Double-membrane vesicles induced by DENV contain NS proteins and dsRNA intermediates, suggesting that they are the site of active RNA synthesis (Miller et al., 2007; Welsch et al., 2009).

Several of the non-structural viral proteins are required to rearrange ER membranes into replication complexes (Schwartz et al., 2002; den Boon and Ahlquist, 2010). Vesicle formation is induced by NS4A through its transmembrane domain (Roosendaal et al., 2006; Miller et al., 2007) and cleavage of its $2 \mathrm{~K}$ peptide induces membrane arrangement (Miller et al., 2007). Additionally, NS4A triggers rearrangement and phosphorylation of vimentin filaments to support DENV replication complex (Teo and Chu, 2014). NS3 has helicase activity to unfold dsRNA during RNA synthesis, while NS5 has both RNAdependent-RNA polymerase and methyltransferase activity used to synthetize and cap RNA, respectively. ER-anchored NS4B binds the NS3/NS2B complex which together with NS5 supports replication. NS2A transmembrane protein is also essential for RNA synthesis and is involved in the replication complex organization (Xie et al., 2014).

\section{Assembly and Maturation Through Endomembrane Network}

Flavivirus assembly occurs within the ER membrane (Figure 7). The capsid protein binds the positive-strand genome at multiple sites to fold and tightly package viral RNA (Pong et al., 2011). Encapsidated RNA is probably released from replication complexes through the existing pores, while the mechanism of transport to assembly sites remains unclear. During assembly, ER-anchored membrane and envelope proteins assemble around the capsid, forming a lipid bilayer (Tan et al., 2020). Importantly, the capsid decorates LDs originating from the ER and harnesses them for assembly (Samsa et al., 2009).

Upon assembly, flaviviruses form immature particles characterized by spikes of trimeric pr-membrane, which is the membrane protein precursor, and envelope proteins (Zhang et al., 2003). Maturation occurs through Golgi and trans-Golgi networks and requires an acidic environment 
(Oliveira et al., 2017; Figure 7). Lower pH induces molecular rearrangement of pr-membrane and envelope proteins to expose pr-membrane. Host furin protease then cleaves pr from pr-membrane, maintaining the membrane protein in the mature virion. The presence of $\mathrm{pr}$ in viral particles during the maturation might prevent the fusion of the viral envelope with the trans-Golgi network (Yu et al., 2009). Immature or partially immature virions with altered infectivity are also produced.

\section{Exocytosis of Virus Particles}

The contents of endomembrane vesicles, i.e., virions and pr peptides, are released into the extracellular space by exocytosis through interaction with the plasma membrane (Figure 7). Little is known about the mechanism of flavivirus egress (Barrows et al., 2018). However, unlike replication and assembly sites in the ER that contain multiple flaviviral particles (Welsch et al., 2009), secretory vesicles usually contain individual flaviviral particles, which are then released individually (Burlaud-Gaillard et al., 2014).

\section{FLAVIVIRUSES SUBDUE PHOSPHOLIPIDS IN MOSQUITOES}

\section{Specificity of Lipid Metabolism in Mosquitoes}

The lipid metabolism pathways are well-conserved between mammals and insects. Fatty acid, PL and glycerolipid biosyntheses are similar except for differences in numbers of enzyme isoforms (Gondim et al., 2018). For instance, while mammals have two acetyl-CoA carboxylases involved in fatty acid metabolism, insects only have one (Alabaster et al., 2011). Similarity in enzyme subcellular localizations, membrane and endomembrane compositions also suggest a certain homology in lipid biosyntheses (Butters and Hughes, 1981). Aedes mosquito cells contain an abundance of PC and PE and the same PL categories as in mammals (Townsend et al., 1972; Jenkin et al., 1975). However, while PC is the main PL in mammalian cells, Aedes mosquito cells contain a majority of PE (Luukkonen et al., 1973; Jenkin et al., 1975; Dawaliby et al., 2016), and other substantial differences in lipid metabolism between insects and mammals exist (Canavoso et al., 2001).

Insects are auxotrophic for cholesterol and must obtain it as well as essential fatty acids from their diet (Figure 8). For mosquitoes, ingested blood is an important source of lipids as it contains free fatty acids, triacylglycerol, cholesterol, and cellassociated PLs. In whole human blood, the most abundant fatty acids are $\mathrm{C} 16: 0, \mathrm{C} 18: 1$, and $\mathrm{C} 18: 2$, while $\mathrm{PC}$ represents $70-$ $72 \%$ of PL in the plasma, $30-36 \%$ in erythrocytes, $35-40 \%$ in thrombocytes (Hodson et al., 2008). Following blood feeding, midgut lipases and phospholipases catabolize lipids and PLs, respectively, to generate fatty acids. Fatty acids are then absorbed by midgut cells and integrated in the PA pathway to produce PL, triacylglycerol and DAG. Fatty acids can also be synthesized from glucose and amino acids, which is significant given the mosquito mixed diet on nectar sugar and high-protein blood. Lipids are transported in Aedes aegypti mosquitoes as TAG in association with the insect lipoprotein called lipophorin, which shuttles lipids to fat body for conversion in triacylglycerol for lipid storage. Stored lipids can be mobilized using the reusable lipophorin shuttle and delivered to targeted tissues for energy or metabolic processes (Gondim et al., 2018). Interestingly, lipophorin is also involved in the immune response and is regulated upon infection (Cheon et al., 2006).

It should be noted that mosquito blood feeding is usually carried out multiple times to reach repletion (Farjana and Tuno, 2013). These multiple blood intakes can successively modify mosquito lipid metabolism. Moreover, blood composition, and more specifically the fatty acid composition of lipids, varies with diet, age, gender, genetic background, and health status (Hodson et al., 2008). As the blood source is an important parameter for the mosquito lipid profile, blood-induced metabolic changes vary among host donors (Kaczmarek et al., 2021). A lipidome study from field-collected mosquitoes is likely to result in high variability, which could explain part of the variation in mosquito vector competence.

\section{Phospholipid Reconfiguration}

Recent studies, including ours, showed that DENV perturb lipid composition in Aedes cell lines, midguts, and whole mosquitoes (Perera et al., 2012; Chotiwan et al., 2018; Vial et al., 2019, 2020). Unfortunately, to our knowledge there is no study with other flavivirus species in mosquitoes. While studies at different tissue levels are complementary, variations in metabolic changes between cells and mosquitoes were observed. In Ae. aegypti mosquito cell line, DENV infection decreased lipid intermediates such as fatty acid and monoacylglycerol, and increased concentrations in unsaturated PC and PS species at $48 \mathrm{~h}$ post infection (Vial et al., 2019). In Aedes albopictus cell line, sphingolipids, PC, lysoPC, lysoPE increased at $36 \mathrm{~h}$ post-infection in infected cells and in endomembrane fractions containing DENV replication complexes (Perera et al., 2012). A majority of upregulated PC species had unsaturated fatty acyl chains, likely synthetized from PL remodeling (Wang and Tontonoz, 2019), which contributes to PL recycling by incorporating polyunsaturated fatty acids. Interestingly, lysoPLs, resulting from PL hydrolysis, were highly increased early in infected cells (Chotiwan et al., 2018; Vial et al., 2019). Because lysoPLs are the first step in PL remodeling, this is consistent with PL remodeling playing a role in $\mathrm{PL}$ reconfiguration during infection. Of note, $\mathrm{PE}$, which represents the majority of PL in insects and is involved in membrane curvature, was not regulated in global cell extracts and only increased in endomembrane fractions (Perera et al., 2012). Such discrepancy between whole cells and subcellular fractions reveals that infection-induced regulation is orchestrated at a fine scale. Overall, these few studies of lipids in cells evidence that flavivirus infection induces a complex reconfiguration of lipid membrane metabolism.

Dengue virus infection in Ae. aegypti midgut also modulated membrane lipids by upregulating PC, PE, PS, PG, lysoPL, lysoPI, mono-, di-, triacylglycerols, and sphingolipids (Chotiwan et al., 2018). In another study, our group observed an elevation of lipid 


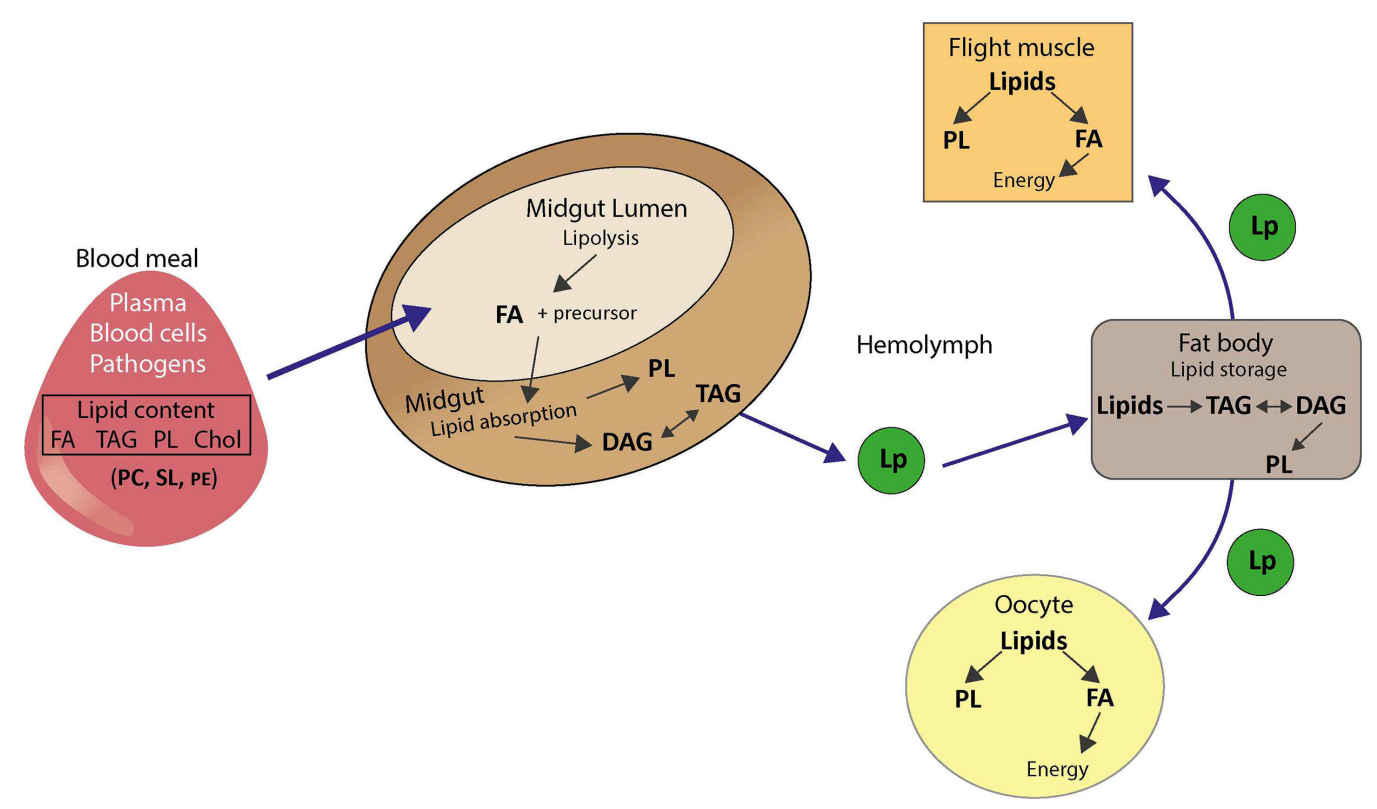

FIGURE 8 | Lipid metabolism in mosquitoes. FA, fatty acid; TAG, triacylglycerol; DAG, diacylglycerol; PL, phospholipid; Cho, cholesterol; PC, phosphatidylcholine; PE, phosphatidylethanolamine; SL, sphingolipid; Lp, lipophorin.

intermediates, PE and PC in infected midguts (Vial et al., 2019), confirming that DENV infection modulates PLs. In the same line of thoughts, our study revealed an early increase in PA, the central intermediate in PL biogenesis (Vial et al., 2019). Inversely, anionic PS had a different behavior in our study as the previous one, as we observed a decrease. Differences in virus and mosquito genetics and physiological status (i.e., nutrition, temperature. ..) may result in metabolic differences. In the sole study with whole mosquitoes, we observed an increase in several species of PLs, especially lysoPL, early in infection, followed by a decrease as infection progressed (Vial et al., 2019). In conclusion, strong modulation of lipids with functions in architecture and expansion of membranes is observed in DENV-infected mosquitoes.

\section{Regulation of de novo Phospholipid Biogenesis}

Phospholipid reconfiguration can occur either by regulating de novo PL biogenesis or PL remodeling, each pathway generating a different set of PLs with different biochemical properties. De novo PLs are generally less unsaturated than remodeled PLs (Barelli and Antonny, 2016). Several lines of evidence indicate that de novo PLs are not favorable to virus multiplication (Figure 9 and Table 1). We have shown that inhibition of several enzymes involved in de novo PL biogenesis promotes DENV infection in mosquito cells (Vial et al., 2020). Likewise, we showed that depletion of AGPAT1 enzyme involved in the synthesis of PA, the precursor for all de novo PLs, increased virus load (Vial et al., 2019). Mosquito AGPATs were phylogenetically characterized based on functional motifs defining substrates affinity and acyltransferase activity (Yamashita et al., 2007) as compared to human AGPATs (Vial et al., 2019). Interestingly, DENV reduced mosquito AGPAT1 expression in vitro and in vivo throughout the infection cycle, whereas another AGPAT isoform with similar function in PA generation was not regulated. Altogether, these results suggest that flaviviruses specifically regulate enzymes of PL biogenesis to promote viral multiplication.

Enzyme expression can be regulated either directly by transcription factor or indirectly by alteration of PL content, ER stress response or endoplasmic reticulum topology. Transcriptionally, multiple enzymes involved in lipid metabolism are regulated by the sterol regulatory element binding proteins (SREBP; Coleman and Lee, 2004; Karasawa et al., 2019). SREBP chemical inhibition was shown to reduce viral replication (Merino-Ramos et al., 2017), suggesting a proviral function for SREBP lipid regulation. However, DENV-induced ER membrane rearrangement was not linked to SREBP signaling (Peña and Harris, 2012). Instead, ER modification was dependent on viral protein expression and lipid reabsorption into the ER. Those results suggest that DENV alteration of PL metabolism is not mediated by SREBP signaling but instead is directly related to recycling of $\mathrm{PL}$ species and viral proteins.

Introgression of the viral proteins into the ER membrane should alter membrane topology, which in turn regulates transmembrane protein function (Bogdanov et al., 2014). For instance, stresses in the lipid bilayer disturb ER-resident proteins (Shyu et al., 2019) and results in accumulation of unfolded or misfolded proteins, a phenomenon known as the unfolded protein response (Gentile et al., 2010). Subsequently, ERinduced stress disrupts lipid metabolism, such as glycerolipid and cholesterol biosynthesis (Werstuck et al., 2001). All these suggest that ER membrane stress imposed by infectious processes might affect enzyme expression and activity by altering 


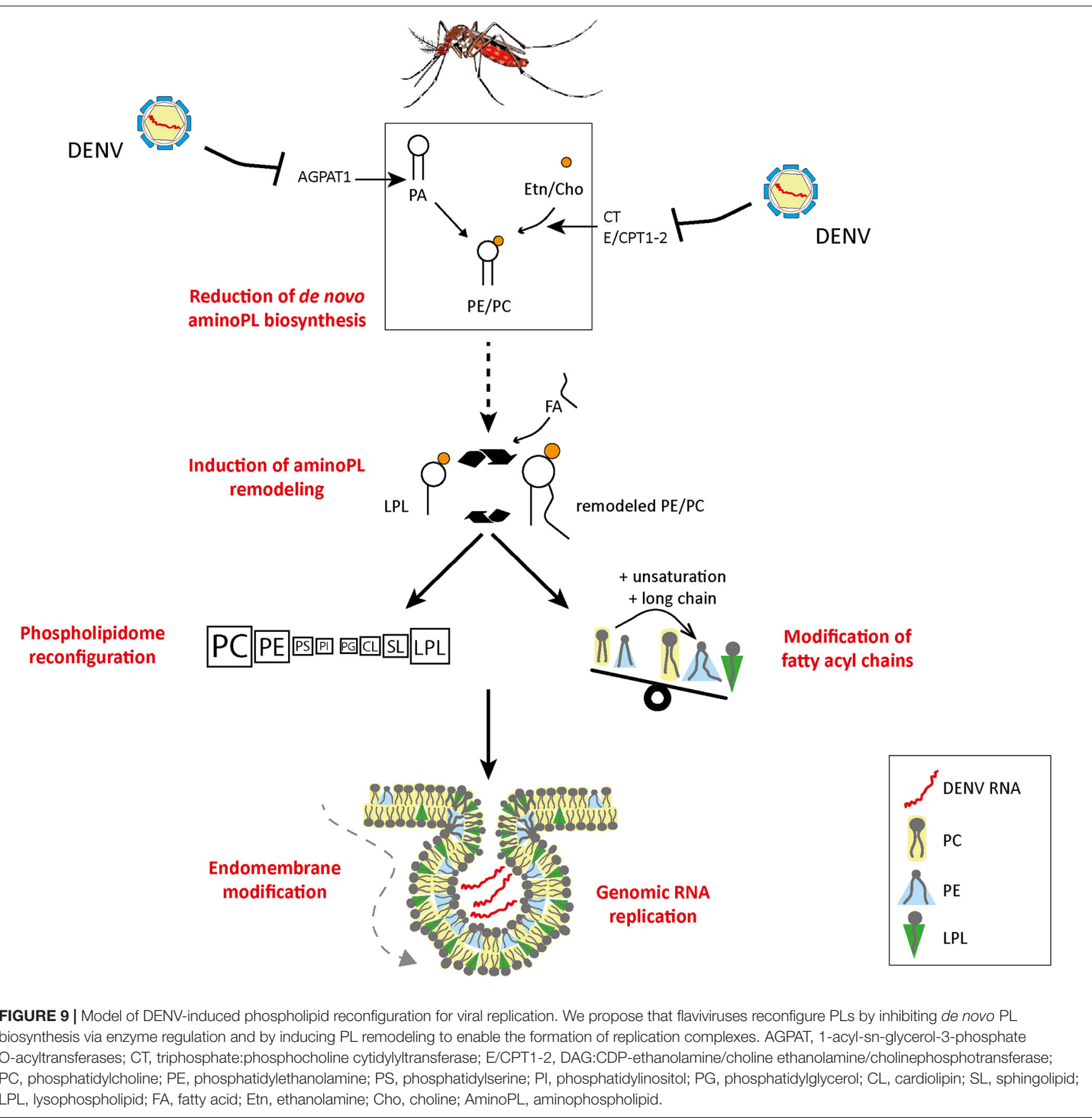

protein anchorage, acyl substrate specificity or transferase activity. These changes to the lipid metabolism may then regulate PL production.

\section{Induction of Phospholipid Remodeling}

The fatty acyl chain of de novo PL can be modified by PL remodeling through the Lands cycle (Figure 5). Remodeling enables cells to produce new PL species with different biochemical properties to support membrane maintenance and diversity (Wang and Tontonoz, 2019). There is a negative crosstalk between de novo PL biogenesis and PL remodeling, whereby activation of one inhibits the other. Previous observation that de novo PL production is inactivated by DENV suggests that PL remodeling is induced by infection (Figure 9; Vial et al., 2020). To demonstrate activation of PL remodeling, we developed isotope tracing of PL in mosquito cells. Cells were supplemented with a labeled precursor of de novo PLs. We provided the label enough time before infection so that PLs but not lysoPLs were labeled. In these conditions, production of lysoPLs indicated PL deacylation and increase in nonlabeled PLs was a consequence of PL remodeling. Inversely, increase in labeled PLs showed activation of de novo PL 
TABLE 1 | Functions of lipid-related mosquito factors in flavivirus infection.

\begin{tabular}{|c|c|c|c|c|}
\hline Pathway & Factor & $\begin{array}{l}\text { Effect on } \\
\text { flavivirus } \\
\text { multiplication }\end{array}$ & $\begin{array}{l}\text { Lipid species } \\
\text { involved } \\
\text { n }\end{array}$ & References \\
\hline \multicolumn{2}{|c|}{ De novo PLFAS } & Proviral & $\begin{array}{l}\mathrm{PL}, \mathrm{SL}, \mathrm{FA} \text { and } \\
\text { intermediates }\end{array}$ & $\begin{array}{l}\text { Perera et al., } \\
2012\end{array}$ \\
\hline & AGPAT1 & Antiviral & PL and LysoPL & Vial et al., 2019 \\
\hline & $\begin{array}{l}\text { Kennedy } \\
\text { pathway }(C T \text {, } \\
\text { ECPT1-2) }\end{array}$ & Antiviral & AminoPL and lysoPL & Vial et al., 2020 \\
\hline & Ethanolamine & Antiviral & AminoPL and LysoPL & Vial et al., 2020 \\
\hline & CL synthase & Proviral & $\mathrm{CL}$ & Koh et al., 2020 \\
\hline $\begin{array}{l}\mathrm{PL} \\
\text { remodeling }\end{array}$ & PLA2 & Proviral & LysoPC & $\begin{array}{l}\text { Liebscher et al., } \\
2018\end{array}$ \\
\hline \multirow[t]{5}{*}{$\begin{array}{l}\text { Other lipid } \\
\text { pathways }\end{array}$} & SL desaturase & Proviral & SL & $\begin{array}{l}\text { Chotiwan et al., } \\
2018\end{array}$ \\
\hline & Lipid droplets & Proviral & $\begin{array}{l}\text { Likely TAG, FA, PL, } \\
\text { sterol esters }\end{array}$ & $\begin{array}{l}\text { Barletta et al., } \\
2016\end{array}$ \\
\hline & $\begin{array}{l}\text { Lipophorin } \\
\text { receptor (LpR) }\end{array}$ & Antiviral & TAG & Koh et al., 2020 \\
\hline & LRP-1 & Antiviral & Cholesterol & $\begin{array}{l}\text { Tree et al., } \\
2019\end{array}$ \\
\hline & SREBP & Proviral & Unknown & $\begin{array}{l}\text { Raquin et al., } \\
2017\end{array}$ \\
\hline
\end{tabular}

FAS, fatty acid synthase; AGPAT1, 1-acyl-sn-glycerol-3-phosphate O-acyltransferase 1; CT, triphosphate:phosphocholine cytidylyltransferase; ECPT, DAG:CDP-ethanolamine/choline ethanolamine/cholinephosphotransferase; Etn, Ethanolamine; PLA2, Phospholipase A2; CL, cardiolipin synthase; DEGS, sphingolipid $\Delta-4$ desaturase; $L D$, lipid droplet; $L p R$, lipophorin receptor; $L R P-1$, low-density lipoprotein receptor-related protein 1; SREBP, sterol regulatory element-binding protein.

biosynthesis. Upon infection, we observed an early production of lysoPLs and an increase in non-labeled PLs, demonstrating induction of the PL remodeling cycle upon infection. However, we subsequently observed an increase in isotope labeled PLs, showing activation of de novo PL biosynthesis. Together, this indicates that DENV infection induces PL remodeling and, later on, de novo PL biogenesis, possibly to replenish PL depleted by remodeling.

Interestingly, in mosquito cells, WNV was shown to increase PLA2 activity (Table 1; Liebscher et al., 2018). PLA2 initiates $\mathrm{PL}$ remodeling by cleaving PL sn2 acyl to produce lysoPLs. Furthermore, the same study showed that lysoPCs were recruited to replication complexes and required for proper membrane curvature in mammalian cells. In a different study, fatty acid synthase enzyme involved in lipogenesis was required during DENV infection in mammalian cells (Perera et al., 2012). DENV NS3 recruited the fatty acid synthase to replication sites and modified the enzyme activity to stimulate malonylCoA incorporation into fatty acids (Heaton et al., 2010). This altered activity produces more palmitate, which is then used for complex lipid biogenesis, including polyunsaturated fatty acids. The newly synthesized fatty acids could serve to reacylate the lysoPLs and complete PL remodeling cycle, thereby diversifying PL composition (The British Nutrition Foundation, 1992). Taken together, we propose a model whereby $\mathrm{PL}$ remodeling is induced by flavivirus infection to generate a membrane architecture conducive to the formation of replication complexes (Figure 9).

\section{Functions of Other Lipid Pathways in Flavivirus Infection}

Dengue virus infection in mosquitoes also relies on other lipids that PLs (Table 1). Sphingolipids are lipid membranes and contribute to DENV multiplication. Chemical inhibition of sphingolipid $\Delta-4$ desaturase, which synthesizes ceramide, reduced DENV multiplication (Chotiwan et al., 2018). Storage of triacylglycerol in LDs is increased upon DENV infection in cells and mosquito midguts (Barletta et al., 2016; Koh et al., 2020). DENV NS4A interacts with Ancient Ubiquitous Protein 1 (AUP1), a LD-associated acyltransferase, to activate LD lipophagy (Zhang et al., 2018). Cholesterol pathway is involved in DENV multiplication (Raquin et al., 2017; Tree et al., 2019). Low-density lipoprotein receptor-related protein 1 (LRP-1) involved in cholesterol regulation was downregulated by infection (Tree et al., 2019), while LRP-1 inhibition increased intracellular cholesterol quantity and promoted DENV infection. A lipophorin receptor (LpR), named as a very low density lipophorin receptor was also down-regulated by DENV infection, although LpR knockdown did not affect DENV replication (Koh et al., 2020). CL synthase involved in CL biogenesis, a mitochondrial PL, was downregulated in DENV-infected Ae. aegypti, although CL synthase promoted infection (Koh et al., 2020). CL alteration may disrupt mitochondrial membrane integrity, modifying intrinsic properties of mitochondria, such as energy metabolism and apoptosis regulation (Gonzalvez and Gottlieb, 2007). Altogether, the whole lipid environment is engaged by flaviviruses to enable viral multiplication.

\section{Phospholipids in Innate Immunity and Signaling}

Mosquitoes mount a potent antiviral response to infection through multiple immune pathways. The Janus kinase/signal transducers and transcription activator pathway (JAK-STAT), immune deficiency pathway (IMD), the Toll pathway, the JunN-terminal Kinase (JNK) pathway, and the RNA interference pathway (RNAi) have all been shown to reduce flavivirus infection (Sim et al., 2014; Chowdhury et al., 2020). Interestingly, there exist bridges in signaling pathways between immune responses and lipid metabolism in mosquitoes. Toll pathway activation by bacteria, fungi or parasites induces expression of fat body genes related to lipid metabolism (Cheon et al., 2006). DENV infection in Ae. aegypti upregulates genes associated with LD biosynthesis, while activation of either Toll or IMD pathways increase LD number (Barletta et al., 2016). The interaction between immune response and lipid metabolism certainly deserves further studies.

Reconfiguration of the lipidome upon infection in mosquitoes may also modify lipid-mediated signaling. Prostaglandins that regulate immunity and inflammation (Harris et al., 2002) are regulated by bacterial and parasite infection in mosquitoes (Muñoz et al., 2008). Prostaglandins are produced from C20 
polyunsaturated fatty acids, which can be derived from PL hydrolysis via PLA2 cleavage during PL remodeling.

\section{Identification of Antiviral Targets}

Because of the lack of treatment and of effective vaccines against all flaviviruses, there is a great need for new strategies to block flavivirus transmission. Targeting branches of lipid metabolism that are required for virus infection may hold the key to novel antiviral tools. While lipid-related anti-flaviviral strategies have already been reviewed (Martín-Acebes et al., 2016), targeting of $\mathrm{PL}$ remains limited when applied to mosquitoes. In mammalian cells, the most preponderant PI (20:4/18:0) is composed of one acyl chain of arachidonic acid and one of stearic acid and has antiDENV activity (Sanaki et al., 2019). The PI acts by suppressing DENV-induced cytokine inflammation.

As PL remodeling promotes DENV infection in mosquitoes (Vial et al., 2020) whereas de novo PL biogenesis reduces virus load, disrupting PL biosynthesis may hinder viral multiplication. Induction of de novo PL biogenesis by supplementing the blood on which mosquitoes feed with a de novo PL precursor reduced mosquito midgut infection (Vial et al., 2020). Harnessing this knowledge, the PL precursor could be delivered through sugar feeding to reduce infection. Similarly, as mosquitoes can derive ethanolamine from $\mathrm{PE}$, de novo $\mathrm{PL}$ biogenesis could be activated by exogenous supply of PE. Moreover, flavivirus infection significantly modifies blood lipid composition (Cui et al., 2013; Lima et al., 2019), which then influences infection onset in mosquito midguts (Vial et al., 2020). Chemical alteration of blood lipids from patients may help disrupt the flavivirus transmission cycle.

Lipid-targeted therapeutics have been identified for other viruses. Influenza virus infection is blocked by the fatty acid docosahexaenoic acid, which limits transport of viral transcripts (Morita et al., 2013). Supplementation with PL such as PG

\section{REFERENCES}

Abdul Rahim, N. A., Othman, M., Sabri, M., and Stanley, D. W. (2018). A Midgut Digestive Phospholipase A2 in Larval Mosquitoes, Aedes albopictus and Culex quinquefasciatus. Enzyme Res. 2018:9703413. doi: 10.1155/2018/9703413

Acosta, E. G., Castilla, V., and Damonte, E. B. (2008). Functional entry of dengue virus into Aedes albopictus mosquito cells is dependent on clathrin-mediated endocytosis. J. Gen. Virol. 89, 474-484. doi: 10.1099/vir.0.83357-0

Acosta, E. G., Castilla, V., and Damonte, E. B. (2011). Infectious dengue-1 virus entry into mosquito C6/36 cells. Virus Res. 160, 173-179. doi: 10.1016/j.virusres. 2011.06.008

Alabaster, A., Isoe, J., Zhou, G., Lee, A., Murphy, A., Day, W. A., et al. (2011). Deficiencies in acetyl-CoA carboxylase and fatty acid synthase 1 differentially affect eggshell formation and blood meal digestion in Aedes aegypti. Insect Biochem. Mol. Biol. 41, 946-955. doi: 10.1016/j.ibmb.2011.09.004

Alkan, C., Zapata, S., Bichaud, L., Moureau, G., Lemey, P., Firth, A. E., et al. (2015). Ecuador Paraiso Escondido Virus, a New Flavivirus Isolated from New World Sand Flies in Ecuador, Is the First Representative of a Novel Clade in the Genus Flavivirus. J. Virol. 89, 11773-11785. doi: 10.1128/JVI.01543-15

Aloulou, A., Rahier, R., Arhab, Y., Noiriel, A., and Abousalham, A. (2018). "Phospholipases: An Overview," in Lipases and Phospholipases: Methods and Protocols Methods in Molecular Biology, ed. G. Sandoval (New York, NY: Springer), 69-105. doi: 10.1007/978-1-4939-8672-9_3

Barelli, H., and Antonny, B. (2016). Lipid unsaturation and organelle dynamics. Curr. Opin. Cell Biol. 41, 25-32. doi: 10.1016/j.ceb.2016.03.012 and PI also suppresses influenza virus and syncytial virus infection. Antibody targeting of PS was applied to treat arenavirus and cytomegalovirus infection in animal models (Soares et al., 2008). Antibodies aimed at PL to induce an immune response were similarly applied to neutralize HIV-1 in peripheral blood mononuclear cells (Moody et al., 2010).

\section{CONCLUSION}

Our understanding of the lipid interactions between mosquitoes and flaviviruses has expanded in recent years, thanks to innovative in vitro and in vivo metabolomic approaches. However, there remain much to understand. The importance of $\mathrm{PL}$ and its reconfiguration for DENV infection in mosquitoes is well established now but studies with multiple flavivirus species are required to determine the conservation of these lipid needs. Further mechanistic analyses will also help identify the PL species involved and understand how flaviviruses alter their concentrations. In the end, characterized PLs will provide ideal targets for novel transmission blocking strategies.

\section{AUTHOR CONTRIBUTIONS}

TV and JP wrote the original draft. TV, DM, GM, and JP edited the manuscript. All authors contributed to the article and approved the submitted version.

\section{FUNDING}

JP received funding from the Agence Nationale de la Recherche (ANR-20-CE15-0006).

Barletta, A. B. F., Alves, L. R., Nascimento Silva, M. C. L., Sim, S., Dimopoulos, G., Liechocki, S., et al. (2016). Emerging role of lipid droplets in Aedes aegypti immune response against bacteria and Dengue virus. Sci. Rep. 6:19928. doi: $10.1038 /$ srep 19928

Barrows, N. J., Campos, R. K., Liao, K.-C., Prasanth, K. R., Soto-Acosta, R., Yeh, S.-C., et al. (2018). Biochemistry and Molecular Biology of Flaviviruses. Chem. Rev. 118, 4448-4482. doi: 10.1021/acs.chemrev.7b00719

Bartz, R., Li, W.-H., Venables, B., Zehmer, J. K., Roth, M. R., Welti, R., et al. (2007). Lipidomics reveals that adiposomes store ether lipids and mediate phospholipid traffic. J. Lipid Res. 48, 837-847. doi: 10.1194/jlr.M600413-JLR200

Beermann, C., Möbius, M., Winterling, N., Schmitt, J. J., and Boehm, G. (2005). sn-Position determination of phospholipid-linked fatty acids derived from erythrocytes by liquid chromatography electrospray ionization ion-trap mass spectrometry. Lipids 40, 211-218. doi: 10.1007/s11745-005-1377-1

Bell, R. M., Ballas, L. M., and Coleman, R. A. (1981). Lipid topogenesis. J. Lipid Res. 22, 391-403.

Bigay, J., and Antonny, B. (2012). Curvature, Lipid Packing, and Electrostatics of Membrane Organelles: Defining Cellular Territories in Determining Specificity. Dev. Cell 23, 886-895. doi: 10.1016/j.devcel.2012.10.009

Bogdanov, M., Dowhan, W., and Vitrac, H. (2014). Lipids and topological rules governing membrane protein assembly. Biochim. Biophys. Acta BBA - Mol. Cell Res. 1843, 1475-1488. doi: 10.1016/j.bbamcr.2013.12.007

Bond, L. M., Miyazaki, M., O’Neill, L. M., Ding, F., and Ntambi, J. M. (2016). "Chapter 6 - Fatty Acid Desaturation and Elongation in Mammals," in Biochemistry of Lipids, Lipoproteins and Membranes (Sixth Edition), eds N. D. 
Ridgway and R. S. McLeod (Boston: Elsevier), 185-208. doi: 10.1016/B978-0444-63438-2.00006-7

Burke, J. E., and Dennis, E. A. (2009). Phospholipase A2 structure/function, mechanism, and signaling. J. Lipid Res. 50, S237-S242. doi: 10.1194/jlr. R800033-JLR200

Burlaud-Gaillard, J., Sellin, C., Georgeault, S., Uzbekov, R., Lebos, C., Guillaume, J.-M., et al. (2014). Correlative Scanning-Transmission Electron Microscopy Reveals that a Chimeric Flavivirus Is Released as Individual Particles in Secretory Vesicles. PLoS One 9:e93573. doi: 10.1371/journal.pone.0093573

Butters, T. D., and Hughes, R. C. (1981). Phospholipids and glycolipids in subcellular fractions of mosquito Aedes aegypti cells. In Vitro 17, 831-838. doi: 10.1007/BF02618451

Byers, N. M., Fleshman, A. C., Perera, R., and Molins, C. R. (2019). Metabolomic Insights into Human Arboviral Infections: Dengue, Chikungunya, and Zika Viruses. Viruses 11:225. doi: 10.3390/v11030225

Canavoso, L. E., Jouni, Z. E., Karnas, K. J., Pennington, J. E., and Wells, M. A. (2001). Fat metabolism in insects. Annu. Rev. Nutr. 21, 23-46. doi: 10.1146/ annurev.nutr.21.1.23

Carnec, X., Meertens, L., Dejarnac, O., Perera-Lecoin, M., Hafirassou, M. L., Kitaura, J., et al. (2016). The Phosphatidylserine and Phosphatidylethanolamine Receptor CD300a Binds Dengue Virus and Enhances Infection. J. Virol. 90, 92-102. doi: 10.1128/JVI.01849-15

Carro, A. C., and Damonte, E. B. (2013). Requirement of cholesterol in the viral envelope for dengue virus infection. Virus Res. 174, 78-87. doi: 10.1016/j. virusres.2013.03.005

Cheon, H.-M., Shin, S. W., Bian, G., Park, J.-H., and Raikhel, A. S. (2006). Regulation of Lipid Metabolism Genes, Lipid Carrier Protein Lipophorin, and Its Receptor during Immune Challenge in the Mosquito Aedes aegypti. J. Biol. Chem. 281, 8426-8435. doi: 10.1074/jbc.M510957200

Chotiwan, N., Andre, B. G., Sanchez-Vargas, I., Islam, M. N., Grabowski, J. M., Hopf-Jannasch, A., et al. (2018). Dynamic remodeling of lipids coincides with dengue virus replication in the midgut of Aedes aegypti mosquitoes. PLoS Pathog. 14:1006853. doi: 10.1371/journal.ppat.1006853

Chowdhury, A., Modahl, C. M., Tan, S. T., Xiang, B. W. W., Missé, D., Vial, T., et al. (2020). JNK pathway restricts DENV2, ZIKV and CHIKV infection by activating complement and apoptosis in mosquito salivary glands. PLoS Pathog. 16:e1008754. doi: 10.1371/journal.ppat.1008754

Coleman, R. A., and Lee, D. P. (2004). Enzymes of triacylglycerol synthesis and their regulation. Prog. Lipid Res. 43, 134-176. doi: 10.1016/S0163-7827(03) 00051-1

Cook, H. W., and McMaster, C. R. (2002). "Chapter 7 Fatty acid desaturation and chain elongation in eukaryotes," in New Comprehensive Biochemistry Biochemistry of Lipids, Lipoproteins and Membranes, 4th Edn, (Amsterdam: Elsevier), 181-204. doi: 10.1016/S0167-7306(02)36009-5

Cruz-Oliveira, C., Freire, J. M., Conceição, T. M., Higa, L. M., Castanho, M. A. R. B., and Da Poian, A. T. (2015). Receptors and routes of dengue virus entry into the host cells. FEMS Microbiol. Rev. 39, 155-170. doi: 10.1093/femsre/fuu004

Cui, L., Lee, Y. H., Kumar, Y., Xu, F., Lu, K., Ooi, E. E., et al. (2013). Serum Metabolome and Lipidome Changes in Adult Patients with Primary Dengue Infection. PLoS Negl. Trop. Dis. 7:e2373. doi: 10.1371/journal.pntd.0002373

Dawaliby, R., Trubbia, C., Delporte, C., Noyon, C., Ruysschaert, J.-M., Antwerpen, P. V., et al. (2016). Phosphatidylethanolamine Is a Key Regulator of Membrane Fluidity in Eukaryotic Cells. J. Biol. Chem. 291, 3658-3667. doi: 10.1074/jbc. M115.706523

den Boon, J. A., and Ahlquist, P. (2010). Organelle-Like Membrane Compartmentalization of Positive-Strand RNA Virus Replication Factories. Annu. Rev. Microbiol. 64, 241-256. doi: 10.1146/annurev.micro.112408.134012

Di Paolo, G., and De Camilli, P. (2006). Phosphoinositides in cell regulation and membrane dynamics. Nature 443, 651-657. doi: 10.1038/nature05185

Ducharme, N. A., and Bickel, P. E. (2008). Minireview: Lipid Droplets in Lipogenesis and Lipolysis. Endocrinology 149, 942-949. doi: 10.1210/en.20071713

Fadeel, B., and Xue, D. (2009). The ins and outs of phospholipid asymmetry in the plasma membrane: roles in health and disease. Crit. Rev. Biochem. Mol. Biol. 44, 264-277. doi: 10.1080/10409230903193307

Fagone, P., and Jackowski, S. (2009). Membrane phospholipid synthesis and endoplasmic reticulum function. J. Lipid Res. 50, S311-S316. doi: 10.1194/jlr. R800049-JLR200
Farjana, T., and Tuno, N. (2013). Multiple Blood Feeding and Host-Seeking Behavior in Aedes aegypti and Aedes albopictus (Diptera: Culicidae). J. Med. Entomol. 50, 838-846. doi: 10.1603/ME12146

Franz, A. W. E., Kantor, A. M., Passarelli, A. L., and Clem, R. J. (2015). Tissue Barriers to Arbovirus Infection in Mosquitoes. Viruses 7, 3741-3767. doi: 10. 3390/v7072795

García Cordero, J., León Juárez, M., González-Y-Merchand, J. A., Cedillo Barrón, L., and Gutiérrez Castañeda, B. (2014). Caveolin-1 in Lipid Rafts Interacts with Dengue Virus NS3 during Polyprotein Processing and Replication in HMEC-1 Cells. PLoS One 9:90704. doi: 10.1371/journal.pone.0090704

Gentile, C. L., Frye, M., and Pagliassotti, M. J. (2010). Endoplasmic Reticulum Stress and the Unfolded Protein Response in Nonalcoholic Fatty Liver Disease. Antioxid. Redox Signal. 15, 505-521. doi: 10.1089/ars.2010.3790

Gibellini, F., and Smith, T. K. (2010). The Kennedy pathway-De novo synthesis of phosphatidylethanolamine and phosphatidylcholine. IUBMB Life 62, 414-428. doi: 10.1002/iub.337

Gondim, K. C., Atella, G. C., Pontes, E. G., and Majerowicz, D. (2018). Lipid metabolism in insect disease vectors. Insect Biochem. Mol. Biol. 101, 108-123. doi: 10.1016/j.ibmb.2018.08.005

Gonzalvez, F., and Gottlieb, E. (2007). Cardiolipin: setting the beat of apoptosis. Apoptosis Int. J. Program. Cell Death 12, 877-885. doi: 10.1007/s10495-0070718-8

Gottlieb, D., Heideman, W., and Saba, J. D. (1999). TheDPL1Gene Is Involved in Mediating the Response to Nutrient Deprivation in Saccharomyces cerevisiae. Mol. Cell Biol. Res. Commun. 1, 66-71. doi: 10.1006/mcbr.1999.0109

Halstead, S. B. (2007). Dengue. Lancet 370, 1644-1652. doi: 10.1016/S01406736(07)61687-0

Halstead, S. B. (2017). Dengvaxia sensitizes seronegatives to vaccine enhanced disease regardless of age. Vaccine 35, 6355-6358. doi: 10.1016/j.vaccine.2017. 09.089

Hardy, J. L., Houk, E. J., Kramer, L. D., and Reeves, W. C. (1983). Intrinsic factors affecting vector competence of mosquitoes for arboviruses. Annu. Rev. Entomol. 28, 229-262. doi: 10.1146/annurev.en.28.010183.001305

Harris, S. G., Padilla, J., Koumas, L., Ray, D., and Phipps, R. P. (2002). Prostaglandins as modulators of immunity. Trends Immunol. 23, 144-150. doi: 10.1016/s1471-4906(01)02154-8

Heaton, N. S., Perera, R., Berger, K. L., Khadka, S., LaCount, D. J., Kuhn, R. J., et al. (2010). Dengue virus nonstructural protein 3 redistributes fatty acid synthase to sites of viral replication and increases cellular fatty acid synthesis. Proc. Natl. Acad. Sci. 107, 17345-17350. doi: 10.1073/pnas.1010811107

Henne, M. L., and Goodman, J. M. (2019). The assembly of lipid droplets and their roles in challenged cells. EMBO J. 38:e101816. doi: 10.15252/embj.201910 1816

Henneberry, A. L., Wright, M. M., and McMaster, C. R. (2002). The Major Sites of Cellular Phospholipid Synthesis and Molecular Determinants of Fatty Acid and Lipid Head Group Specificity. Mol. Biol. Cell 13, 3148-3161. doi: 10.1091/mbc. 01-11-0540

Hidari, K. I. P. J., and Suzuki, T. (2011). Dengue virus receptor. Trop. Med. Health 39, 37-43. doi: 10.2149/tmh.2011-S03

Hodson, L., Skeaff, C. M., and Fielding, B. A. (2008). Fatty acid composition of adipose tissue and blood in humans and its use as a biomarker of dietary intake. Prog. Lipid Res. 47, 348-380. doi: 10.1016/j.plipres.2008.03.003

Holthuis, J. C. M., and Levine, T. P. (2005). Lipid traffic: floppy drives and a superhighway. Nat. Rev. Mol. Cell Biol. 6, 209-220. doi: 10.1038/nrm1591

Hope, M. J., and Cullis, P. R. (1987). Lipid asymmetry induced by transmembrane pH gradients in large unilamellar vesicles. J. Biol. Chem. 262, 4360-4366. doi: 10.1016/S0021-9258(18)61356-0

Horvath, S. E., Böttinger, L., Vögtle, F.-N., Wiedemann, N., Meisinger, C., Becker, T., et al. (2012). Processing and Topology of the Yeast Mitochondrial Phosphatidylserine Decarboxylase 1. J. Biol. Chem. 287, 36744-36755. doi: 10. 1074/jbc.M112.398107

Horvath, S. E., and Daum, G. (2013). Lipids of mitochondria. Prog. Lipid Res. 52, 590-614. doi: 10.1016/j.plipres.2013.07.002

Huang, C.-H., Tsai, Y.-T., Wang, S.-F., Wang, W.-H., and Chen, Y.-H. (2021). Dengue vaccine: an update. Expert Rev. Anti Infect. Ther. 2021, 1-8. doi: 10. 1080/14787210.2021.1949983

Jackson, C. L. (2019). Lipid droplet biogenesis. Curr. Opin. Cell Biol. 59, 88-96. doi: 10.1016/j.ceb.2019.03.018 
Janmey, P. A., and Kinnunen, P. K. J. (2006). Biophysical properties of lipids and dynamic membranes. Trends Cell Biol. 16, 538-546. doi: 10.1016/j.tcb.2006.08. 009

Jenkin, H. M., McMeans, E., Anderson, L. E., and Yang, T.-K. (1975). Comparison of phospholipid composition of Aedes aegypti and Aedes albopictus cells obtained from logarithmic and stationary phases of growth. Lipids 10, 686-694. doi: $10.1007 / \mathrm{BF} 02532762$

Jouhet, J. (2013). Importance of the hexagonal lipid phase in biological membrane organization. Front. Plant Sci. 4:494. doi: 10.3389/fpls.2013.00494

Junjhon, J., Pennington, J. G., Edwards, T. J., Perera, R., Lanman, J., and Kuhn, R. J. (2014). Ultrastructural Characterization and Three-Dimensional Architecture of Replication Sites in Dengue Virus-Infected Mosquito Cells. J. Virol. 88, 4687-4697. doi: 10.1128/JVI.00118-14

Kaczmarek, A., Wrońska, A. K., Boguś, M. I., Kazek, M., Gliniewicz, A., Mikulak, E., et al. (2021). The type of blood used to feed Aedes aegypti females affects their cuticular and internal free fatty acid (FFA) profiles. PLoS One 16:e0251100. doi: 10.1371/journal.pone.0251100

Karasawa, K., Tanigawa, K., Harada, A., and Yamashita, A. (2019). Transcriptional Regulation of Acyl-CoA:Glycerol-sn-3-Phosphate Acyltransferases. Int. J. Mol. Sci. 20:20040964. doi: 10.3390/ijms20040964

Kobayashi, T., Beuchat, M.-H., Chevallier, J., Makino, A., Mayran, N., Escola, J.-M., et al. (2002). Separation and Characterization of Late Endosomal Membrane Domains. J. Biol. Chem. 277, 32157-32164. doi: 10.1074/jbc.M20283 8200

Kobayashi, T., and Menon, A. K. (2018). Transbilayer lipid asymmetry. Curr. Biol. 28, R386-R391. doi: 10.1016/j.cub.2018.01.007

Koh, C., Islam, M. N., Ye, Y. H., Chotiwan, N., Graham, B., Belisle, J. T., et al. (2020). Dengue virus dominates lipid metabolism modulations in Wolbachiacoinfected Aedes aegypti. Commun. Biol. 3:1254. doi: 10.1038/s42003-02001254-z

Kolter, T., and Sandhoff, K. (2005). PRINCIPLES OF LYSOSOMAL MEMBRANE DIGESTION: Stimulation of Sphingolipid Degradation by Sphingolipid Activator Proteins and Anionic Lysosomal Lipids. Annu. Rev. Cell Dev. Biol. 21,81-103. doi: 10.1146/annurev.cellbio.21.122303.120013

Krahmer, N., Guo, Y., Wilfling, F., Hilger, M., Lingrell, S., Heger, K., et al. (2011). Phosphatidylcholine Synthesis for Lipid Droplet Expansion Is Mediated by Localized Activation of CTP:Phosphocholine Cytidylyltransferase. Cell Metab. 14, 504-515. doi: 10.1016/j.cmet.2011.07.013

Lazear, H. M., and Diamond, M. S. (2016). Zika Virus: New Clinical Syndromes and Its Emergence in the Western Hemisphere. J. Virol. 90, 4864-4875. doi: 10.1128/JVI.00252-16

Lee, C.-J., Lin, H.-R., Liao, C.-L., and Lin, Y.-L. (2008). Cholesterol Effectively Blocks Entry of Flavivirus. J. Virol. 82, 6470-6480. doi: 10.1128/JVI.00117-08

Leier, H. C., Messer, W. B., and Tafesse, F. G. (2018). Lipids and pathogenic flaviviruses: An intimate union. PLoS Pathog. 14:e1006952. doi: 10.1371/journal. ppat. 1006952

Leventis, P. A., and Grinstein, S. (2010). The Distribution and Function of Phosphatidylserine in Cellular Membranes. Annu. Rev. Biophys. 39, 407-427. doi: 10.1146/annurev.biophys.093008.131234

Li, J., Wang, X., Zhang, T., Wang, C., Huang, Z., Luo, X., et al. (2015). A review on phospholipids and their main applications in drug delivery systems. Asian J. Pharm. Sci. 10, 81-98. doi: 10.1016/j.ajps.2014.09.004

Liebscher, S., Ambrose, R. L., Aktepe, T. E., Mikulasova, A., Prier, J. E., Gillespie, L. K., et al. (2018). Phospholipase A2 activity during the replication cycle of the flavivirus West Nile virus. PLoS Pathog. 14:1007029. doi: 10.1371/journal.ppat. 1007029

Lima, W. G., Souza, N. A., Fernandes, S. O. A., Cardoso, V. N., and Godói, I. P. (2019). Serum lipid profile as a predictor of dengue severity: A systematic review and meta-analysis. Rev. Med. Virol. 29:2056. doi: 10.1002/rmv.2056

Lin, D. L., Inoue, T., Chen, Y.-J., Chang, A., Tsai, B., and Tai, A. W. (2019). The ER Membrane Protein Complex Promotes Biogenesis of Dengue and Zika Virus Non-structural Multi-pass Transmembrane Proteins to Support Infection. Cell Rep. 27, 1666.e-1674.e. doi: 10.1016/j.celrep.2019.04.051

Lu, Y., and Chen, C. (2017). Metabolomics: Bridging Chemistry and Biology in Drug Discovery and Development. Curr. Pharmacol. Rep. 3, 16-25. doi: 10. 1007/s40495-017-0083-4

Lundbæk, J. A. (2006). Regulation of membrane protein function by lipid bilayer elasticity - a single molecule technology to measure the bilayer properties experienced by an embedded protein. J. Phys. Condens. Matter 18, S1305-S1344. doi: 10.1088/0953-8984/18/28/S13

Luukkonen, A., Brummer-Korvenkontio, M., and Renkonen, O. (1973). Lipids of cultured mosquito cells (Aedes albopictus): Comparison with cultured mammalian fibroblasts (BHK 21 cells). Biochim. Biophys. Acta BBA - Lipids Lipid Metab. 326, 256-261. doi: 10.1016/0005-2760(73)90251-8

Ma, L., Jones, C. T., Groesch, T. D., Kuhn, R. J., and Post, C. B. (2004). Solution structure of dengue virus capsid protein reveals another fold. Proc. Natl. Acad. Sci. 101, 3414-3419. doi: 10.1073/pnas.0305892101

Manchester, M., and Anand, A. (2017). "Chapter Two - Metabolomics: Strategies to Define the Role of Metabolism in Virus Infection and Pathogenesis," in Advances in Virus Research, eds M. Kielian, T. C. Mettenleiter, and M. J. Roossinck (Cambridge, MA: Academic Press), 57-81. doi: 10.1016/bs.aivir. 2017.02.001

Marquardt, D., Geier, B., and Pabst, G. (2015). Asymmetric Lipid Membranes: Towards More Realistic Model Systems. Membranes 5, 180-196. doi: 10.3390/ membranes5020180

Marsh, D. (2007). Lateral Pressure Profile, Spontaneous Curvature Frustration, and the Incorporation and Conformation of Proteins in Membranes. Biophys. J. 93, 3884-3899. doi: 10.1529/biophysj.107.107938

Martens, C., Shekhar, M., Borysik, A. J., Lau, A. M., Reading, E., Tajkhorshid, E., et al. (2018). Direct protein-lipid interactions shape the conformational landscape of secondary transporters. Nat. Commun. 9:4151. doi: 10.1038/ s41467-018-06704-1

Martín-Acebes, M. A., Merino-Ramos, T., Blázquez, A.-B., Casas, J., EscribanoRomero, E., Sobrino, F., et al. (2014). The Composition of West Nile Virus Lipid Envelope Unveils a Role of Sphingolipid Metabolism in Flavivirus Biogenesis. J. Virol. 88, 12041-12054. doi: 10.1128/JVI.02061-14

Martín-Acebes, M. A., Vázquez-Calvo, Á, and Saiz, J.-C. (2016). Lipids and flaviviruses, present and future perspectives for the control of dengue, Zika, and West Nile viruses. Prog. Lipid Res. 64, 123-137. doi: 10.1016/j.plipres.2016.09. 005

Mayr, J. A. (2015). Lipid metabolism in mitochondrial membranes. J. Inherit. Metab. Dis. 38, 137-144. doi: 10.1007/s10545-014-9748-x

McMahon, H. T., and Boucrot, E. (2015). Membrane curvature at a glance. J. Cell Sci. 128:1065. doi: 10.1242/jcs. 114454

Meertens, L., Carnec, X., Lecoin, M. P., Ramdasi, R., Guivel-Benhassine, F., Lew, E., et al. (2012). The TIM and TAM Families of Phosphatidylserine Receptors Mediate Dengue Virus Entry. Cell Host Microbe 12, 544-557. doi: 10.1016/j. chom.2012.08.009

Merino-Ramos, T., Jiménez, de Oya, N., Saiz, J.-C., and Martín-Acebes, M. A. (2017). Antiviral Activity of Nordihydroguaiaretic Acid and Its Derivative Tetra-O-Methyl Nordihydroguaiaretic Acid against West Nile Virus and Zika Virus. Antimicrob. Agents Chemother. 61:17. doi: 10.1128/AAC.00376-17

Miller, S., Kastner, S., Krijnse-Locker, J., Bühler, S., and Bartenschlager, R. (2007). The Non-structural Protein 4A of Dengue Virus Is an Integral Membrane Protein Inducing Membrane Alterations in a 2K-regulated Manner. J. Biol. Chem. 282, 8873-8882. doi: 10.1074/jbc.M609919200

Moessinger, C., Klizaite, K., Steinhagen, A., Philippou-Massier, J., Shevchenko, A., Hoch, M., et al. (2014). Two different pathways of phosphatidylcholine synthesis, the Kennedy Pathway and the Lands Cycle, differentially regulate cellular triacylglycerol storage. BMC Cell Biol. 15:43. doi: 10.1186/s12860-0140043-3

Moody, M. A., Liao, H.-X., Alam, S. M., Scearce, R. M., Plonk, M. K., Kozink, D. M., et al. (2010). Anti-phospholipid human monoclonal antibodies inhibit CCR5-tropic HIV-1 and induce $\beta$-chemokines. J. Exp. Med. 207, 763-776. doi: $10.1084 /$ jem. 20091281

Morgan, C. P., Insall, R., Haynes, L., and Cockcroft, S. (2004). Identification of phospholipase B from Dictyostelium discoideum reveals a new lipase family present in mammals, flies and nematodes, but not yeast. Biochem. J. 382, 441-449. doi: 10.1042/BJ20040110

Morita, M., Kuba, K., Ichikawa, A., Nakayama, M., Katahira, J., Iwamoto, R., et al. (2013). The Lipid Mediator Protectin D1 Inhibits Influenza Virus Replication and Improves Severe Influenza. Cell 153, 112-125. doi: 10.1016/j.cell.2013.02. 027

Mosso, C., Galván-Mendoza, I. J., Ludert, J. E., and del Angel, R. M. (2008). Endocytic pathway followed by dengue virus to infect the mosquito cell line C6/36 HT. Virology 378, 193-199. doi: 10.1016/j.virol.2008.05.012 
Muñoz, F. L. G. G., de Martínez-Barnetche, J., Lanz-Mendoza, H., Rodríguez, M. H., and Hernández-Hernández, F. C. (2008). Prostaglandin E2 modulates the expression of antimicrobial peptides in the fat body and midgut of Anopheles albimanus. Arch. Insect Biochem. Physiol. 68, 14-25. doi: 10.1002/ arch.20232

Ngo, A. M., Shurtleff, M. J., Popova, K. D., Kulsuptrakul, J., Weissman, J. S., and Puschnik, A. S. (2019). The ER membrane protein complex is required to ensure correct topology and stable expression of flavivirus polyproteins. eLife 8:48469. doi: $10.7554 /$ eLife.48469

Nor Aliza, A. R., and Stanley, D. W. (1998). A digestive phospholipase A2 in larval mosquitoes, Aedes aegypti. Insect Biochem. Mol. Biol. 28, 561-569. doi: 10.1016/S0965-1748(98)00050-2

Nunes, E. C., and Canuto, G. A. B. (2020). Metabolomics applied in the study of emerging arboviruses caused by Aedes aegypti mosquitoes: A review. Electrophoresis 41, 2102-2113. doi: 10.1002/elps.202000133

O'Donnell, V. B., Rossjohn, J., and Wakelam, M. J. O. (2018). Phospholipid signaling in innate immune cells. J. Clin. Invest. 128, 2670-2679. doi: 10.1172/ JCI97944

Oliveira, E. R. A., de Alencastro, R. B., and Horta, B. A. C. (2017). New insights into flavivirus biology: the influence of $\mathrm{pH}$ over interactions between prM and E proteins. J. Comput. Aided Mol. Des. 31, 1009-1019. doi: 10.1007/s10822-0170076-8

O’Neal, A. J., Butler, L. R., Rolandelli, A., Gilk, S. D., and Pedra, J. H. (2020). Lipid hijacking: a unifying theme in vector-borne diseases. eLife 9:e61675. doi: 10.7554/eLife.61675

Onono, F. O., and Morris, A. J. (2020). Phospholipase D and Choline Metabolism. Handb. Exp. Pharmacol. 259, 205-218. doi: 10.1007/164_2019_320

Ooi, E.-E., Goh, K.-T., and Gubler, D. J. (2006). Dengue Prevention and 35 Years of Vector Control in Singapore. Emerg. Infect. Dis. J. 12:6. doi: 10.3201/eid1206. 051210

Parikh, G. R., Oliver, J. D., and Bartholomay, L. C. (2009). A haemocyte tropism for an arbovirus. J. Gen. Virol. 90, 292-296. doi: 10.1099/vir.0.005116-0

Patton-Vogt, J., and de Kroon, A. I. P. M. (2020). Phospholipid turnover and acyl chain remodeling in the yeast ER. Biochim. Biophys. Acta Mol. Cell Biol. Lipids 1865:158462. doi: 10.1016/j.bbalip.2019.05.006

Peña, J., and Harris, E. (2012). Early Dengue Virus Protein Synthesis Induces Extensive Rearrangement of the Endoplasmic Reticulum Independent of the UPR and SREBP-2 Pathway. PLoS One 7:38202. doi: 10.1371/journal.pone. 0038202

Penno, A., Hackenbroich, G., and Thiele, C. (2013). Phospholipids and lipid droplets. Biochim. Biophys. Acta BBA - Mol. Cell Biol. Lipids 1831, 589-594. doi: 10.1016/j.bbalip.2012.12.001

Perera, R., Riley, C., Isaac, G., Hopf-Jannasch, A. S., Moore, R. J., Weitz, K. W., et al. (2012). Dengue Virus Infection Perturbs Lipid Homeostasis in Infected Mosquito Cells. PLoS Pathog. 8:1002584. doi: 10.1371/journal.ppat.100 2584

Pierson, T. C., and Diamond, M. S. (2020). The continued threat of emerging flaviviruses. Nat. Microbiol. 5, 796-812. doi: 10.1038/s41564-020-0714-0

Pong, W.-L., Huang, Z.-S., Teoh, P.-G., Wang, C.-C., and Wu, H.-N. (2011). RNA binding property and RNA chaperone activity of dengue virus core protein and other viral RNA-interacting proteins. FEBS Lett. 585, 2575-2581. doi: 10.1016/ j.febslet.2011.06.038

Radhakrishnan, A., Goldstein, J. L., McDonald, J. G., and Brown, M. S. (2008). Switch-like control of SREBP-2 transport triggered by small changes in ER cholesterol: a delicate balance. Cell Metab. 8, 512-521. doi: 10.1016/j.cmet.2008. 10.008

Raquin, V., Merkling, S. H., Gausson, V., Moltini-Conclois, I., Frangeul, L., Varet, H., et al. (2017). Individual co-variation between viral RNA load and gene expression reveals novel host factors during early dengue virus infection of the Aedes aegypti midgut. PLoS Negl. Trop. Dis. 11:6152. doi: 10.1371/journal.pntd. 0006152

Raturi, A., and Simmen, T. (2013). Where the endoplasmic reticulum and the mitochondrion tie the knot: The mitochondria-associated membrane (MAM). Biochim. Biophys. Acta BBA - Mol. Cell Res. 1833, 213-224. doi: 10.1016/j. bbamcr.2012.04.013

Reddy, T., and Sansom, M. S. P. (2016). The Role of the Membrane in the Structure and Biophysical Robustness of the Dengue Virion Envelope. Structure 24, 375-382. doi: 10.1016/j.str.2015.12.011
Richard, A. S., Zhang, A., Park, S.-J., Farzan, M., Zong, M., and Choe, H. (2015). Virion-associated phosphatidylethanolamine promotes TIM1-mediated infection by Ebola, dengue, and West Nile viruses. Proc. Natl. Acad. Sci. 112, 14682-14687. doi: 10.1073/pnas. 1508095112

Rivers, J. P. W., Sinclair, A. J., and Crawford, M. A. (1975). Inability of the cat to desaturate essential fatty acids. Nature 258, 171-173. doi: 10.1038/258171a0

Roosendaal, J., Westaway, E. G., Khromykh, A., and Mackenzie, J. M. (2006). Regulated Cleavages at the West Nile Virus NS4A-2K-NS4B Junctions Play a Major Role in Rearranging Cytoplasmic Membranes and Golgi Trafficking of the NS4A Protein. J. Virol. 80, 4623-4632. doi: 10.1128/JVI.80.9.4623-4632. 2006

Rusiñol, A. E., Cui, Z., Chen, M. H., and Vance, J. E. (1994). A unique mitochondria-associated membrane fraction from rat liver has a high capacity for lipid synthesis and contains pre-Golgi secretory proteins including nascent lipoproteins. J. Biol. Chem. 269, 27494-27502. doi: 10.1016/S0021-9258(18) 47012-3

Salazar, M. I., Richardson, J. H., Sánchez-Vargas, I., Olson, K. E., and Beaty, B. J. (2007). Dengue virus type 2: replication and tropisms in orally infected Aedes aegypti mosquitoes. BMC Microbiol. 7:9. doi: 10.1186/1471-2180-7-9

Samsa, M. M., Mondotte, J. A., Iglesias, N. G., Assunção-Miranda, I., BarbosaLima, G., Da Poian, A. T., et al. (2009). Dengue Virus Capsid Protein Usurps Lipid Droplets for Viral Particle Formation. PLoS Pathog. 5:1000632. doi: 10. 1371/journal.ppat.1000632

Sanaki, T., Wakabayashi, M., Yoshioka, T., Yoshida, R., Shishido, T., Hall, W. W., et al. (2019). Inhibition of dengue virus infection by 1-stearoyl-2-arachidonoylphosphatidylinositol in vitro. FASEB J. 33, 13866-13881. doi: 10.1096/fj. 201901095RR

Schwartz, M., Chen, J., Janda, M., Sullivan, M., den Boon, J., and Ahlquist, P. (2002). A Positive-Strand RNA Virus Replication Complex Parallels Form and Function of Retrovirus Capsids. Mol. Cell 9, 505-514. doi: 10.1016/S10972765(02)00474-4

Shukla, R., Ramasamy, V., Shanmugam, R. K., Ahuja, R., and Khanna, N. (2020). Antibody-Dependent Enhancement: A Challenge for Developing a Safe Dengue Vaccine. Front. Cell. Infect. Microbiol. 10:572681. doi: 10.3389/fcimb.2020. 572681

Shyu, P., Ng, B. S. H., Ho, N., Chaw, R., Seah, Y. L., Marvalim, C., et al. (2019). Membrane phospholipid alteration causes chronic ER stress through early degradation of homeostatic ER-resident proteins. Sci. Rep. 9, 1-15. doi: 10.1038/ s41598-019-45020-6

Sim, S., Jupatanakul, N., and Dimopoulos, G. (2014). Mosquito Immunity against Arboviruses. Viruses 6, 4479-4504. doi: 10.3390/v6114479

Soares, M. M., King, S. W., and Thorpe, P. E. (2008). Targeting Inside-Out Phosphatidylserine as a Therapeutic Strategy For Viral Diseases. Nat. Med. 14, 1357-1362. doi: 10.1038/nm.1885

Sul, H. S., and Smith, S. (2008). "CHAPTER 6 - Fatty acid synthesis in eukaryotes," in Biochemistry of Lipids, Lipoproteins and Membranes (Fifth Edition), eds D. E. Vance and J. E. Vance (San Diego: Elsevier), 155-190. doi: 10.1016/B978044453219-0.50008-8

Takeuchi, K., and Reue, K. (2009). Biochemistry, physiology, and genetics of GPAT, AGPAT, and lipin enzymes in triglyceride synthesis., Biochemistry, physiology, and genetics of GPAT, AGPAT, and lipin enzymes in triglyceride synthesis. Am. J. Physiol. Endocrinol. Metab. Am. J. Physiol. - Endocrinol. Metab. 1195, E1195-E1209. doi: 10.1152/ajpendo.90958.2008

Tamura, Y., Onguka, O., Itoh, K., Endo, T., Iijima, M., Claypool, S. M., et al. (2012). Phosphatidylethanolamine biosynthesis in mitochondria: phosphatidylserine (PS) trafficking is independent of a PS decarboxylase and intermembrane space proteins UPS1P and UPS2P. J. Biol. Chem. 287, 43961-43971. doi: 10.1074/jbc. M112.390997

Tan, T. Y., Fibriansah, G., Kostyuchenko, V. A., Ng, T.-S., Lim, X.-X., Zhang, S., et al. (2020). Capsid protein structure in Zika virus reveals the flavivirus assembly process. Nat. Commun. 11:895. doi: 10.1038/s41467-020-14647-9

Teo, C. S. H., and Chu, J. J. H. (2014). Cellular vimentin regulates construction of dengue virus replication complexes through interaction with NS4A protein. J. Virol. 88, 1897-1913. doi: 10.1128/JVI.01249-13

The British Nutrition Foundation (1992). "Metabolism of Unsaturated Fatty Acids," in Unsaturated Fatty Acids: Nutritional and physiological significance: The Report of the British Nutrition Foundation's Task Force, (Boston, MA: Springer), 35-47. doi: 10.1007/978-1-4899-4429-0_7 
Thiam, A. R., and Forêt, L. (2016). The physics of lipid droplet nucleation, growth and budding. Biochim. Biophys. Acta BBA - Mol. Cell Biol. Lipids 1861, 715-722. doi: 10.1016/j.bbalip.2016.04.018

Townsend, D., Jenkin, H. M., and Tze-Ken, Y. (1972). Lipid analysis of Aedes aegypti cells cultivated in vitro. Biochim. Biophys. Acta BBA - Lipids Lipid Metab. 260, 20-25. doi: 10.1016/0005-2760(72)90069-0

Tree, M. O., Londono-Renteria, B., Troupin, A., Clark, K. M., Colpitts, T. M., and Conway, M. J. (2019). Dengue virus reduces expression of low-density lipoprotein receptor-related protein 1 to facilitate replication in Aedes aegypti. Sci. Rep. 9, 6352. doi: 10.1038/s41598-019-42803-9

van Meer, G., Voelker, D. R., and Feigenson, G. W. (2008). Membrane lipids: where they are and how they behave. Nat. Rev. Mol. Cell Biol. 9, 112-124. doi: $10.1038 / \mathrm{nrm} 2330$

Vance, D. E. (2013). Physiological roles of phosphatidylethanolamine N-methyltransferase. Biochim. Biophys. Acta BBA - Mol. Cell Biol. Lipids 1831, 626-632. doi: 10.1016/j.bbalip.2012.07.017

Vance, D. E., Li, Z., and Jacobs, R. L. (2007). Hepatic Phosphatidylethanolamine N-Methyltransferase, Unexpected Roles in Animal Biochemistry and Physiology. J. Biol. Chem. 282, 33237-33241. doi: 10.1074/jbc.R700028200

Vance, D. E., and Vance, J. E. (2008). "CHAPTER 8 - Phospholipid biosynthesis in eukaryotes," in Biochemistry of Lipids, Lipoproteins and Membranes (Fifth Edition), eds D. E. Vance and J. E. Vance (San Diego: Elsevier), 213-244. doi: 10.1016/B978-044453219-0.50010-6

Vance, J. E. (2015). Phospholipid Synthesis and Transport in Mammalian Cells. Traffic 16, 1-18. doi: 10.1111/tra.12230

Vial, T., Tan, W.-L., Deharo, E., Missé, D., Marti, G., and Pompon, J. (2020). Mosquito metabolomics reveal that dengue virus replication requires phospholipid reconfiguration via the remodeling cycle. Proc. Natl. Acad. Sci. 117, 27627-27636. doi: 10.1073/pnas.2015095117

Vial, T., Tan, W.-L., Xiang, B. W. W., Missé, D., Deharo, E., Marti, G., et al. (2019). Dengue virus reduces AGPAT1 expression to alter phospholipids and enhance infection in Aedes aegypti. PLoS Pathog. 15:e1008199. doi: 10.1371/journal. ppat.1008199

Wang, B., and Tontonoz, P. (2019). Phospholipid Remodeling in Physiology and Disease. Annu. Rev. Physiol. 81, 165-188. doi: 10.1146/annurev-physiol020518-114444

Wang, H., Becuwe, M., Housden, B. E., Chitraju, C., Porras, A. J., Graham, M. M., et al. (2016). Seipin is required for converting nascent to mature lipid droplets. eLife 5:e16582. doi: 10.7554/eLife.16582

Welsch, S., Miller, S., Romero-Brey, I., Merz, A., Bleck, C. K. E., Walther, P., et al. (2009). Composition and three-dimensional architecture of the dengue virus replication and assembly sites. Cell Host Microbe 5, 365-375. doi: 10.1016/j. chom.2009.03.007

Welte, M. A., and Gould, A. P. (2017). Lipid droplet functions beyond energy storage. Biochim. Biophys. Acta 1862, 1260-1272. doi: 10.1016/j.bbalip.2017.0 7.006

Werstuck, G. H., Lentz, S. R., Dayal, S., Hossain, G. S., Sood, S. K., Shi, Y. Y., et al. (2001). Homocysteine-induced endoplasmic reticulum stress causes dysregulation of the cholesterol and triglyceride biosynthetic pathways. J. Clin. Invest. 107, 1263-1273. doi: 10.1172/JCI11596

Williamson, P. (2016). Phospholipid Scramblases. Lipid Insights 8, 41-44. doi: 10.4137/LPI.S31785
Wortmann, S. B., and Mayr, J. A. (2019). Choline-related-inherited metabolic diseases-A mini review. J. Inherit. Metab. Dis. 42, 237-242. doi: 10.1002/jimd. 12011

Xie, X., Zou, J., Puttikhunt, C., Yuan, Z., and Shi, P.-Y. (2014). Two Distinct Sets of NS2A Molecules Are Responsible for Dengue Virus RNA Synthesis and Virion Assembly. J. Virol. 89, 1298-1313. doi: 10.1128/JVI.02882-14

Yamashita, A., Hayashi, Y., Matsumoto, N., Nemoto-Sasaki, Y., Oka, S., Tanikawa, T., et al. (2014). Glycerophosphate/Acylglycerophosphate Acyltransferases. Biology 3, 801-830. doi: 10.3390/biology3040801

Yamashita, A., Nakanishi, H., Suzuki, H., Kamata, R., Tanaka, K., Waku, K., et al. (2007). Topology of acyltransferase motifs and substrate specificity and accessibility in 1-acyl-sn-glycero-3-phosphate acyltransferase 1. Biochim. Biophys. Acta BBA - Mol. Cell Biol. Lipids 1771, 1202-1215. doi: 10.1016/j. bbalip.2007.07.002

Yamashita, S., and Nikawa, J. (1997). Phosphatidylserine synthase from yeast. Biochim. Biophys. Acta BBA - Lipids Lipid Metab. 1348, 228-235. doi: 10.1016/ S0005-2760(97)00102-1

Yan, R., Qian, H., Lukmantara, I., Gao, M., Du, X., Yan, N., et al. (2018). Human SEIPIN Binds Anionic Phospholipids. Dev. Cell 47, 248.e-256.e. doi: 10.1016/j. devcel.2018.09.010

Yu, I.-M., Holdaway, H. A., Chipman, P. R., Kuhn, R. J., Rossmann, M. G., and Chen, J. (2009). Association of the pr Peptides with Dengue Virus at Acidic pH Blocks Membrane Fusion. J. Virol. 83, 12101-12107. doi: 10.1128/JVI.016 37-09

Zaitseva, E., Yang, S.-T., Melikov, K., Pourmal, S., and Chernomordik, L. V. (2010). Dengue Virus Ensures Its Fusion in Late Endosomes Using CompartmentSpecific Lipids. PLoS Pathog. 6:1001131. doi: 10.1371/journal.ppat.100 1131

Zhang, J., Lan, Y., Li, M. Y., Lamers, M. M., Fusade-Boyer, M., Klemm, E., et al. (2018). Flaviviruses Exploit the Lipid Droplet Protein AUP1 to Trigger Lipophagy and Drive Virus Production. Cell Host Microbe 23, 819.e-831.e. doi: 10.1016/j.chom.2018.05.005

Zhang, Y., Corver, J., Chipman, P. R., Zhang, W., Pletnev, S. V., Sedlak, D., et al. (2003). Structures of immature flavivirus particles. EMBO J. 22, 2604-2613. doi: $10.1093 / \mathrm{emboj} / \mathrm{cdg} 270$

Conflict of Interest: The authors declare that the research was conducted in the absence of any commercial or financial relationships that could be construed as a potential conflict of interest.

Publisher's Note: All claims expressed in this article are solely those of the authors and do not necessarily represent those of their affiliated organizations, or those of the publisher, the editors and the reviewers. Any product that may be evaluated in this article, or claim that may be made by its manufacturer, is not guaranteed or endorsed by the publisher.

Copyright (C) 2021 Vial, Marti, Missé and Pompon. This is an open-access article distributed under the terms of the Creative Commons Attribution License (CC BY). The use, distribution or reproduction in other forums is permitted, provided the original author(s) and the copyright owner(s) are credited and that the original publication in this journal is cited, in accordance with accepted academic practice. No use, distribution or reproduction is permitted which does not comply with these terms. 